\title{
A Hierarchical Ensemble of ECOC for Cancer Classification Based on Multi-Class Microarray Data
}

\author{
Kun-Hong Liu ${ }^{1,2, *}$, Zhi-Hao Zeng ${ }^{1}$, Vincent To Yee $\mathrm{Ng}^{2, \dagger}$ \\ 1, the School of Software, Xiamen University \\ ${ }^{2}$ the Department of Computing, The Hong Kong Polytechnic University
}

\begin{abstract}
The difficulty of the cancer classification using multi-class microarray datasets lies in that there are only a few samples in each class. To effectively solve such a problem, we propose a hierarchical ensemble strategy, named as Hierarchical Ensemble of Error Correcting Output Codes (HE-ECOC). In this strategy, different feature subsets extracted from a dataset are used as inputs for three data-dependent ECOC algorithms, so as to produce different ECOC coding matrices. The mutual diversity degrees among these coding matrices are then calculated based on two schemes, named as the maximizing local diversity (MLD) and the maximizing global diversity (MGD) schemes. Both schemes can choose diverse coding matrices generated by the same or different ECOC algorithm(s), and the average fusion scheme is used to fuse the outputs of base learners. In the experiments, it is found that both MLD and MGD based HE-ECOC strategies work stably, and outperform individual single ECOC algorithms. In contrast with some ensemble systems, HE-ECOC generates a more robust ensemble system, and achieves better performance in most case. In short, HE-ECOC is a promising solution for the multi-class problem. The matlab code is available upon request.
\end{abstract}

Keywords: Error Correcting Output Codes (ECOC); ensemble learning; cancer classification; feature selection; multi-class microarray data. 


\section{Introduction}

\subsection{Background}

With the development of microarray technology, it is possible to diagnose and classify some particular cancers directly based on DNA microarray datasets. Up to now, more and more new prediction, classification and clustering techniques are being used for the analysis of the data. For example, Golub et al. utilized a nearest-neighbor classifier method for the classification of acute myeloid lymphoma (AML) and acute leukemia lymphoma (ALL) in children[22]. And some studies have been reported on the application of microarray gene expression data analysis for molecular classification of cancer $[2,46]$. In short, microarray experiments lead to a more complete understanding of the molecular variations among tumors, and hence achieve finer and more reliable classification. Although it provides a gold mine of biological information and knowledge, it brings new challenges for biologists, statisticians and machine learning researchers because the number of tumor samples collected tends to be much smaller than the number of genes. That is, the number for the former tends to be on the order of tens or hundreds, while microarray datasets typically contain thousands of genes on each chip. As it is a typical "large p, small n" problem[47], an efficient and effective method for the gene expression data analysis is still a challenge.

In the field of machine learning and pattern recognition, the goal of a classification algorithm is to search a mapping function: $f: S \rightarrow K$, in which $S$ is a set of samples and $K$ is the corresponding class labels. For a binary class problem, there are already many widely used machine learning algorithms for estimating $f$. However, for a multi-class problem with $N$ classes, a single learner is hard to produce accurate results in most cases. What's more, many elaborate classifiers, such as support vector machine (SVM), can only deal with binary problems by nature. In order to solve a multi-class problem, an alternative is to use the divide and conquer method to divide the original multi-class problem into multiple binary class problems. After dealing with each binary classification problem independently, a multi-class classification task can be handled by combining their outputs with some fusing schemes, such as the majority voting scheme. In this way, the multiclass problem is tackled by a multiple classifier system (MCS), which is proved to be more accurate and robust than an excellent single classifier in many fields[31].

With this idea, there are three basic approaches: flat strategy, hierarchical strategy and Error-Correcting Output Codes (ECOC). For the flat strategy, a fixed decomposition method, such as One vs. One (OVO) or One vs. Rest (OVR), is used, and the final label is decided directly by voting. The hierarchical strategy employs a binary tree to represent a class decomposition scheme. In the tree, each branch node represents a binary classifier and each leaf node represents a class. As for ECOC, it works in two steps: the encoding step and the decoding step. In the encoding step, the original multi-class problem is divided into multiple binary problems, represented by an $N \times M$ coding matrix: $N$ rows represent $N$ classes, and $M$ columns represent a decomposition scheme 
using $M$ dichotomizers. In the decoding step, the outputs of all dichotomizers are compared with each row in the coding matrix, and only the row achieving the minimum distance is selected as the final decision[17]. Hence ECOC can be considered as a more general solution than the flat and hierarchical strategies because all decomposition schemes of the former two strategies can be regarded as some special ECOC coding matrices. In addition, Dietterich and Kong proved that ECOC can reduce bias and variance errors produced by the binary classifiers more effectively [28]. So in this study, our work focuses on the application of ECOC to the field of microarray analysis.

\subsection{Related Work}

The ECOC framework has been successfully applied in a wide range of research fields. In different studies, researchers found that it is a hard job to produce the optimum coding matrix, especially when $N$ is large. So in recent years, many researchers devoted to the study of optimal ECOC algorithms from different perspectives. Different encoding and decoding schemes have been extensively studied. Masulli and Valentini [36] analyzed different factors that affect the effectiveness of ECOC algorithms, and found that the effectiveness of ECOC depends on the correlation between each code-word pair, structure and accuracy of dichotomizers, along with data complexity. Up to now, there is still no universal rule to design an optimal ECOC coding matrix method, and Crammer and Singer [10] proved that the design of ECOC associated to the problem domain is an NP-complete problem. So many researchers tried to use heuristic algorithms in the coding process to obtain compact coding matrix with fewer dichotomizers. It was discussed that the optimum number of dichotomizers is $\left[10 \log _{2} N, 15 \log _{2} N\right][1]$. Bautista et al. [5]proposed Minimal ECOC to optimize ECOC coding matrix using a standard genetic algorithm (GA), and they reduced the number of binary classifiers to $\left[\log _{2} N\right]$ without losing discriminative ability. Garcia-Pedrajas and Fyfe [21] used the CHC based GA to optimize the sparse random coding matrix, the length of which is limited within $[30,50]$ regardless of the distribution of training data. Lorena and Carvalho [34] combined GA with the Sparse Random coding matrix too, and limited the length of code within $\left[\log _{2} N, N\right]$. Furthermore, Miguer and Sergio [16] proposed a new genetic operator to avoid invalid individuals and reduced the search space of the GA. However, these methods are all based on data independent coding methods. It should be noted that the predefined coding schemes, such as OVO, random-based and all aforementioned ECOC schemes, cannot promise the best solutions for classification tasks: all such schemes neglect the data distributions.

To obtain better solutions, many researchers designed different data dependent methods by taking data distributions into consideration. For example, in [4], different feature subsets are applied to train different dichotomizers, so as to make them more independent. Discriminated ECOC (DECOC) [38] method can generate a hierarchical partition of the class space to maximize the difference among classes by using $N-1$ dichotomizers. The partitions of a problem are learned by 
means of a binary tree structure using exhaustive search or a SFFS criterion, and each internal node of the tree is embedded as a column in the coding matrix. Forest-ECOC [15] uses $(N-1) \times T$ dichotomizers, where $T$ stands for the number of binary tree structures to be embedded. It uses the classification score to create each node for building trees, and finds the trees with the maximum scores at each node to construct a robust ensemble system. ECOC-ONE [14] uses a validation set to extend the initial matrix, and increases its generalization ability by including new dichotomizers focusing on the hard classes. And it requires $2 \times N$ dichotomizers.

Although ECOC algorithms have already been well studied, its application to microarray data is just at the beginning. The difficulty of microarray data classification problem lies in the small sample size problem, which makes a validation set unaffordable. In recent years, many methods were proposed to deal with multiclass microarray datasets. For example, a gene pairs based SVM ensemble system was designed to realize the oncogene recognition and cancer classification simultaneously [45]. Yu H. L. et. al used a multiclass SVM to classify multi-class microarray data with the aid of classification confidence measure [48]. And there are also some evolutionary algorithms based ensemble systems proposed to deal with this problem[32, 33]. However, there are no enough explorations in using ECOC to this problem. A typical application of ECOC was carried out in [13]. In that paper, the authors designed a recursive ECOC algorithm based on the LDPC decoding scheme[44], which was applied to tackle the classification and significant gene subsets selection tasks at the same time based on seven microarray data sets.

Unlike the existing ECOC algorithms, we propose a Hierarchical Ensemble of ECOC (HE-ECOC) method by fusing different ECOC coding matrices with two strategies: the maximizing local diversity (MLD) and maximizing global diversity (MGD) strategies. Some experiments are carried out based on multiple microarray datasets, and the experimental results prove that this ensemble method can further improve the discrimination power of the original ECOC methods. Our algorithm is also compared with some other algorithms, verifying the effectiveness of the HE-ECOC algorithm.

The rest of this paper is organized as follows. Section 2 overviews the background of ECOC algorithms. Section 3 presents our HE-ECOC framework, and describes the MLD and MGD schemes in details along with a concrete example. Section 4 is devoted to presenting the experimental results and the corresponding discussions, and Section 5 concludes this paper.

\section{The Background of Error Correcting Output Codes}

Let $K$ denote a set of unique labels, $K=\left\{k_{1}, k_{2}, \ldots, k_{N}\right\}$, where $N$ is the number of classes $(N>$ 2). Let $S$ denote a set of samples, $S=\left\{\left(X_{1}, \mathrm{y}_{1}\right),\left(X_{2}, \mathrm{y}_{2}\right), \ldots .,\left(X_{L}, \mathrm{y}_{L}\right)\right\}$, where $L$ is the number of 
samples, $X_{i}$ is the feature vector representing $S_{i}$, and $y_{i}$ is its class label $\left(y_{i} \in K\right)$. Let $D=\left\{d_{1}, d_{2}, \ldots\right.$, $\left.d_{M}\right\}$ denote a set of dichotomizers. An ECOC coding matrix $E$ consists of $r$ rows and c columns. The basic idea of ECOC is to build a unique code word for each class.

\subsection{The Encoding process}

In the encoding step, the original class label is mapped to a set of binary class labels, and the mapping scheme is recorded in a coding matrix. Different encoding schemes tend to produce different coding matrices, and are basically divided into two categories: the static scheme and the dynamic scheme [4]. The static scheme commonly constructs coding matrices independent of base classifiers and datasets. On the contrary, the dynamic scheme constructs data-dependent coding matrices, so it is much more flexible but complicated compared with the static schemes. In the dynamic schemes, it is important to take row/column separation into consideration. Row separation refers to a distance between any pair of code words, and column separation indicates the diversity among binary classifier pairs. Row/column separation should be as large as possible, so as to reduce the correlation between base classifiers and code words.

After producing the coding matrix, there are some essential rules to check the legality of the matrix [6], as shown in Formula (1-4), where $r^{i} / \mathrm{c}^{i}$ is the $i$-th row/column of the coding matrix. Formula (1) indicates that there should not exist two identical rows in a coding matrix, where AHD represents Attenuated Hamming Distance. Formula (2) shows that the minimum Hamming distance (HD) between two columns should be at least 1. Formula (3) warns that if there is a converse relationship between any two columns, the encoding matrix is invalid. Formula (4) indicates that the number of binary classifiers should be at least $\log _{2} N$. And the validity check provides pseudo integrity protection while constructing coding matrix.

$$
\begin{aligned}
& \min \left(A H D\left(r^{i}, r^{j}\right)\right) \geq 1, \forall i, j: i \neq j ; i, j \in[1, \ldots, N] \\
& \min \left(H D\left(c^{i}, c^{l}\right)\right) \geq 1, \forall i, l: i \neq l ; i, l \in[1, \ldots, M] \\
& \min \left(H D\left(c^{i},-c^{l}\right)\right) \geq 1, \forall i, l: i \neq l ; i, l \in[1, \ldots, M] \\
& M \geq \log _{2} N
\end{aligned}
$$




\subsection{The Decoding process}

When testing a sample $X^{*}$, each binary classifier produces an output, and a group of outputs make up a vector $V^{*}$ with length $M$. Let $V^{* j}$ represents the $j$-th element in $V^{*}$. Then, the distance between the output vector and code words in the coding matrix is calculated. The code word with the minimum distance is selected as the final decision, and the corresponding class label $o$ is assigned to $X^{*}$. This process is called decoding, and there are many different decoding schemes. Among them, hamming decoding is the most commonly used scheme, as shown in Formula (5) and (6). However, it has obvious drawbacks as it requires that each binary classifier only produces hard outputs, +1 or -1 . With Euclidean decoding scheme, this problem can be solved, as shown in Formula (7).

$$
\begin{aligned}
& H D\left(V^{*}, E\left(r_{i}, \bullet\right)\right)=\sum_{j=1}^{M} \frac{\left(1-s i g\left(V^{* j}, E\left(r_{i}, c_{j}\right)\right)\right)}{2} \\
& o=\arg \min _{i=\{1, \ldots, N\}} H D\left(V^{*}, E\left(r_{i}, \bullet\right)\right) \\
& E D\left(V^{*}, E\left(r_{i}, \bullet\right)\right)=\sqrt{\sum_{j=1}^{M}\left(V^{* j}, E\left(r_{i}, c_{j}\right)^{2}\right.}
\end{aligned}
$$

Besides distance based decoding strategies, researchers also proposed some other schemes based on loss function[1]. The loss function is firstly calculated according to the output vector $V^{*}$, as shown in Formula (8). Loss function $L(\theta)$ depends on the characteristics of base classifier, and the most commonly used functions are $L(\theta)=-\theta$ and $L(\theta)=e^{-\theta}$. Then, the code word achieving the minimum loss function value is picked as the class label for the sample. Moreover, probability based decoding schemes have also been proposed by taking probability estimation and confidence into consideration.

$$
L B\left(V^{*}, E\left(r_{i}, \bullet\right)\right)=\sum_{j=1}^{M} L\left(V^{* j}, E\left(r_{i}, c_{j}\right)\right)
$$

\section{The Hierarchical Ensemble of ECOC}

The error correction capability of ECOC coding matrix is one of the most important factors in the design of ECOC methods. It is pointed out that the coding matrix can fix $d$ bits' error if the 
code matrix's minimum hamming distance equals $2 d+1$ [23]. Therefore, many random-based or data-dependent algorithms try to maximize the minimum hamming distance. In addition, the ability of detecting and correcting errors depends on whether the errors occur independently, so it is essential to improve the binary classifiers' mutual independence.

In general, different data-dependent ECOC algorithms can produce diverse coding matrices for a same dataset owing to different principles. Inspired by this observation, we propose an ensemble method based on diverse ECOC coding matrices. In our method, an ECOC algorithm is regarded as a base learner, and the HE-ECOC method fuses a set of ECOC learner to solve the multi-class microarray problem. The framework of HE-ECOC is given in the following sections.

\subsection{The framework of HE-ECOC}

In general, the success of an ensemble system depends on the accurate and diverse base learner. However, due to the small sample size problem in microarray datasets, it is impossible to provide validation sets after training, and there is no way to directly assess the performance of base learners before testing unknown samples. On the other hand, since each ECOC algorithm is already an ensemble system, we can simply consider them as reliable base learners, and mainly concentrate on the diversity among different base learners in building an ensemble system.

The HE-ECOC method consists of two steps: (1) Generating several diverse ECOC coding matrices using some data-dependent ECOC algorithms; (2) Evaluating the local or global diversity for all coding matrices, and fusing the most diverse matrices to construct an ensemble system. In the first step of the HE-ECOC method, a set of ECOC coding matrices are produced. It is obvious that effective candidates are key to the final ensemble system. In order to produce diverse coding matrices, different data subsets are selected as inputs for a/some data-dependent ECOC algorithm(s). The second step involves the design of diversity measures. There are a lot of different diversity measures designed by various principles for evaluating base classifiers in an ensemble system. The most commonly applied ones are based on the difference of the base learners' outputs. However, since the number of training samples is not large for a microarray dataset, overfitting may occur when applying the output-based diversity measures. In order to avoid the problem, we design two diversity measures to evaluate the difference among ECOC 
coding matrices directly, which can be used to safely select diverse coding matrices for the final ensemble system. The measures are implemented in two different schemes: maximizing local diversity (MLD) and maximizing global diversity (MGD).

In the MLD scheme, a feature selection (FS) method is used to select feature subsets with different sizes. Such data subsets are fed to a data-dependent ECOC algorithm, which can then generate a set of different coding matrices. The pairwise diversity $(P D)$ between a coding matrix pair $\left(C_{i}, C_{j}\right)$ is calculated using Formula (9), and the top ranking matrices are combined to form an ensemble system. The two matrices in a pair are assigned to the same $P D$ score. For those matrices obtaining the same $P D$ score, the global pairwise diversity $(G P D)$ will be used as a reference, and the one with a higher GPD score gets a higher rank. The GPD is calculated by measuring the $P D$ scores between one coding matrix and all other matrices, shown as Formula (10), where $N_{S}$ is the number of coding matrices (it also refers to the ensemble size). The pseudo code of MLD is given in Fig 1.

$$
\begin{aligned}
P D_{i, j} & =\sum_{l=1}^{\text {length }\left(C_{i}\right)}\left(\min _{m \in\left\{1, \text { ength }\left(C_{j}\right)\right\} ; c_{l} \in C_{i} ; c_{m} \in C_{j}}\left(H D\left(c_{l}, c_{m}\right)\right)\right) \\
G P D_{i} & =\sum_{j=2}^{N_{S}} P D_{i, j}
\end{aligned}
$$

As for MGD, different ECOC algorithms are applied to construct coding matrices with different feature subsets. Then, the GPD scores are calculated, and the top coding matrices are chosen. Because various ECOC algorithms may produce coding matrices with diverse lengths, the MGD scheme sorts coding matrices according to their lengths firstly. Assume that the length of matrix $C_{i}$ is shorter than that of $C_{j}$. When calculating the $G P D$ score for a matrix pair $C_{i}$ and $C_{j}$, MGD compares a column in $C_{i}$ with all columns in $C_{j}$, and matches it with the one in $C_{j}$ obtaining the shortest Hamming distance. After all columns in $C_{i}$ are checked, the distance between $C_{i}$ and $C_{j}$ is determined by accumulating the Hamming distances of all columns in $C_{i}$. The pseudo code of MGD is given in Fig. 2, with listing only the parameters different from those of MLD. And it is a noteworthy option that MGD scheme can be implemented using a single ECOC algorithm. Since the diversity calculation process of MGD is different from that of MLD, even using the same ECOC learner, different coding matrices may be picked by these two schemes.

The coding matrices selected by MLD/MGD scheme are used to classify multi-class datasets individually. Each ECOC is regarded as a base learner in our method, and a set of ECOC learners 
are fused using the average scheme (AVE for short). That is, the distances of base learners are averaged, and the label obtaining the minimum mean distance is chosen as the final decision. Assume that for a sample $X^{*}$, the $i$-th ECOC base learner produces an output vector $V_{i}^{*}$, and its corresponding Hamming distance for class $j$ is $H D_{i j}$, then the final output for $X^{*}$ is:

$$
o=\arg \min { }_{j=\{1, \ldots, N\}}\left(\sum_{i=1}^{N_{S}} \frac{H D_{i j}}{N_{S}}\right)
$$

With this scheme, the final HE-ECOC system is much more robust than a single ECOC because it produces reliable results. In the experiments discussed in subsection 4.1 and 4.2 , only AVE is used for the fusion of base learners. In order to demonstrate the advantage of the AVE scheme, other different fusion schemes are also used for comparisons. The corresponding results and discussions are given in subsection 4.3.

\subsection{An example of MLD and MGD}

An example of the process of coding matrices selection based on MLD and MGD schemes is provided with on the Breast dataset [37] using Laplacian Scores [24] FS method and Forest-ECOC algorithm. Let $C M_{n}$ represent a coding matrix generated using $n$ genes. In Fig. 3, the axes represent the size of feature subsets used to produce coding matrices, and the number in each unit represents the $P D$ score of a coding matrix pair calculated by Formula (9). For example, the number 16.7 in the second unit of the first row refers to the $P D$ score for $C M_{20}$ and $C M_{40}$. The diversity matrix is shown as an upper triangular matrix, and all units on the main diagonal line of the matrix are all filled with 0 .

When applying the MLD scheme, it can be found in Fig.3 that the maximum $P D$ value is 36.7, and $C M_{180}$ and $C M_{200}$ are ranked as the first and second highest diverse matrix. To identify which one gets the highest score, the $G P D$ scores are calculated by Formula (10). $C M_{180} / C M_{200}$ obtains $261.1 / 213.5$, respectively. So $C M_{180}$ gets the highest rank. The second highest $P D$ score is 35, achieved by $C M_{180}$ and $C M_{100}$. Since $C M_{180}$ is already selected, it won't be chosen again, and $C M_{100}$ is selected as the third highest diverse matrix. The following $P D$ value is 33.3 , therefore $C M_{80}$ and $C M_{140}$ are chosen. Again, by checking their $G D P$ scores, it is found that $C M_{80}$ and $C M_{140}$ score 68.3 and 66.7 , and are ranked as the fourth and fifth, respectively. In this way, when 
using MLD, the ranking of $P D$ scores in descending order is $\left\{C M_{180}, C M_{200}, C M_{100}, C M_{80}, C M_{140}\right.$, $\left.C M_{40}, C M_{120}, C M_{20}, C M_{60}, C M_{160}\right\}$. As a result, when the final ensemble size of the HE-ECOC system is 5, $\left\{C M_{180}, C M_{200}, C M_{100}, C M_{80}, C M_{140}\right\}$ are chosen.

When the MGD scheme is applied, the GPD scores are calculated. To simplify our problem, assume that only an ECOC algorithm is used, so the selection process is still carried out based on the $P D$ score matrix shown in Fig. 3. The $P D$ score between $C M_{i}$ and all other coding matrices is accumulated as $G P D_{i}$, calculated by Formula (10). In this example, $G P D$ scores are $\{175.1,198.5$, $183.4,200,163.4,226.8,194.9,191.7,270.1,213.5\}$ for $C M_{20}$ to $C M_{200}$ in sequence. Therefore, the final ranking is $\left\{C M_{180}, C M_{120}, C M_{200}, C M_{80}, C M_{40}, C M_{140}, C M_{160}, C M_{60}, C M_{20}, C M_{100}\right\}$. When selecting five coding matrices to construct an ensemble system, the MGD scheme chooses $\left\{C M_{180}, C M_{120}, C M_{200}, C M_{80}, C M_{40}\right\}$. In comparison with the results obtained by MLD, it can be found that $C M_{180}, C M_{200}$ and $C M_{80}$ are commonly selected, and two out of five are different. It is obvious that MGD and MLD works for different purposes, so the final outputs tends to be different. In general, MLD focuses on the diversity within a classifier pair, and MGD focuses on the diversity in a whole classifier pool. Since fewer candidate coding matrices are involved in the MLD scheme, the diversity among matrices may be limited. And MGD gets a better chance to pick up more diverse coding matrices in the final ensemble.

\section{Experimental Results and Analysis}

In our experiments, three ECOC methods implemented in ECOC library [18] are employed: DECOC, forest-ECOC, and ECOC-One. Two base classifiers are used: KNN (k=3) and SVM (Lib-SVM library[9]). Hamming distance function is applied for decoding. Default settings are applied for other parameters. There are many different feature selection algorithms designed for microarray datasets [8]. In our work, three feature selection methods are used: Su[49], Laplacian Score [24] and t-test[11]. The feature size increases from 20 to 200 at the step of 20 in each case. In the MGD scheme, three ECOC methods are used at the same time to promote diversity. In the first part of experiments, two cancer datasets are mainly used for comparisons: the Cancers [42] and Breast[37] datasets. And then five other datasets are applied for further discussions. The details about these datasets are given in Table 1. Only the average fusion scheme is used in the experiments 
in subsection 4.1, 4.2 and 4.3.

\subsection{The Comparisons of single ECOC algorithms and HE-ECOC}

Firstly, the performance of a single ECOC method is evaluated. Fig. 4 shows the results obtained by ECOC-One algorithm for the Cancers and Breast datasets. It can be found that the performance of ECOC-One varies greatly with the changes of feature subsets in both datasets, and it is hard to determine how many genes are needed to achieve the best results, which is also the dilemma of other data-dependent ECOC algorithms in the filed of microarray data analysis. It is obvious that ECOC-One cannot always produce accurate results for microarray datasets, which holds true for other ECOC algorithms in our experiments. In short, these single ECOC algorithms cannot perform well in the classification task for microarray datasets.

On the contrary, HE-ECOC achieves higher average accuracies with lower standard variance compared with single ECOC in most experiments. Table 2 and 3 summarize the results on both datasets using different FS methods. Methods (a), (b), and (c) show the average and best results obtained by a single ECOC algorithm. Methods (d), (e), and (f) represent the application of MLD based HE-ECOC using different single ECOC algorithms as base learners. Method (g) represents MGD based HE-ECOC.

From Table 2 and 3, it is observed that with different FS methods, HE-ECOC can produce quite different results, because all ECOC methods are data-dependent and the changes of inputs affect the results greatly. The average accuracy of each single ECOC algorithm is only about $65 \%$ for the Cancers datasets. In case of using KNN, HE-ECOC cannot further improve the performance with Su FS method, and the average and best accuracies are around $60 \%$ for all methods. In contrast, the application of SVM can completely change this situation. Using SVM, HE-ECOC can raise the average result up to $83.40 \%$. As indicated by previous works, accurate base classifiers are required to build an effective ensemble system. For all remaining cases, we can find that the average results obtained by HE-ECOC can usually be improved by $10 \%-20 \%$ compared with every single ECOC method. The improvement rate even reaches $33 \%$ in the case of MLD based HE-ECOC with Forest-ECOC compared with using Forest-ECOC alone for the Cancers dataset with Laplacian Score. It can be found that for both datasets, the best accuracies and best average accuracies are mostly produced by the HE-ECOC algorithms. Furthermore, the 
MLD based HE-ECOC achieves similar performance when compared with the MGD based one, and their performance is stable with low standard variance in most experiments.

Typical results obtained by MLD based HE-ECOC are shown in Fig. 5 and 6. In general, the performance is promoted when fusing more coding matrices, and the accuracy curves may keep relatively steady after fusing three base learners. In a few cases, it is found that when the ensemble size is larger than 5, the performance of HE-ECOC may begin to deteriorate sometimes. The reason is that there are only 10 candidate matrices in our experiments. When more than 5 coding matrices are selected, lower diversity would be unavoidable in the final ensemble.

The classification results obtained by the MGD based HE-ECOC are shown in Fig. 7. Similar observations are made that in most cases. That is, the MGD based HE-ECOC performance becomes better with the number of base ECOC learners increasing, but do not change much when the ensemble size is larger than three. Usually, when the ensemble size reaches 5, satisfying results can be guaranteed.

To further evaluate the effectiveness of our approach, 5 other benchmark microarray datasets are also deployed: SRBCT [27], Lung [25], DLBCL [41], GCM [39], Leukemia [7]. The average accuracies and best accuracies obtained in the experiments are shown in Table 4 and 5 using SVM and t-test as FS method. The results are also obtained by using varying sizes of feature subset. For fair comparisons, only one ECOC algorithm is deployed for MGD in this case. The results based on DECOC and Forest-ECOC are listed for comparisons with setting the ensemble size as 5.

Experimental results confirm the feasibility of our proposed method again. It is obvious that for each dataset, DECOC method can produce results with average accuracies higher than $50 \%$, so both MLD and MGD based HE-ECOC can improve the performance with increasing average accuracy level by more than $10 \%$. For the Leukemia and GCM datasets, the individual ECOC learners cannot tackle the classification tasks well, and only yield the average accuracies around $60 \%$. Despite such weak base learners, our HE-ECOC can still generate promising results. For Forest-ECOC based experiments, it is found that both MLD and MGD based HE-ECOC greatly improve the performance for most datasets. However, for the GCM dataset, Forest-ECOC algorithm is too weak to build qualified learners, and the average accuracies of base learners are lower than $50 \%$, even worse than random guess. As a result, the final ensemble system finds no 
way to produce ideal results. In conclusion, if the base learners can achieve good performances, our HE-ECOC can further improve the performance.

\subsection{The Further Insight of Results}

\section{(A) The mean ranking analysis}

When the mean of the rankings in each experiment is computed, a test can be carried out to check whether there is a statistically significant difference in the mean ranks for each group. The mean ranks for each method are calculated and shown in Table. 6 and 7, which are applied to further compare the performances achieved by single ECOC algorithms and the MLD/MGD based HE-ECOC methods. The rankings are obtained by estimating each particular ranking $r_{i}^{j}$ for each method $i$ in each experiment $j$ for the Breast and Cancers datasets. The mean ranking $R$ is calculated by:

$$
R_{j}=\frac{1}{N} \times \sum_{i} r_{i}^{j}
$$

where $N$ is the total number of different experiments. The mean rankings are calculated based on those of the best accuracies and average accuracies. And the smaller value of the mean ranking, the better performance.

In order to compare the difference of performances using the mean ranking measurement, the Nemenyi test [37] is applied to single out these methods, and the Nemenyi statistic is obtained as follows:

$$
C D=q_{\alpha} \sqrt{\frac{k \times(k+1)}{6 N}}
$$

In our case, for $k=3$ (three methods: Single ECOC, MLD and MGD) and $N=2$ (two datasets), the critical value for a $90 \%$ of confidence interval is:

$$
C D=2.589 \times \sqrt{\frac{3 \times 4}{12}}=2.589
$$

The statistical tests are presented in Fig. 8. The horizontal bar across each square shows the critical difference, and the mean ranking of each method is indicated by a square in the middle of a bar. If two methods are significantly different, their corresponding average rankings would be separated by at least the critical difference value. That is, their horizontal bars would not overlap, and a large value of distance indicates significant difference.

From Table 6, 7 and Fig. 8, it can be safely concluded that both MLD and MGD based 
HE-ECOC beat single ECOC algorithms in considering both the average accuracy and best accuracy rankings. They take obvious advantages over each single ECOC method in performance, especially with elaborate base classifiers. And the relatively short lengths of the bars presenting the MGD/MLD based HE-ECOC show that our method is more stable in experiments. In addition, the overlap of the horizontal bars standing for HE-ECOC methods demonstrates that the performance of MLD based HE-ECOC is close to that of MGD based HE-ECOC.

\section{(B) Comparisons of MLD and MGD Kappa Statistic}

To further explore the diversity among base learners, Kappa statistic is applied to compare the performance of MLD and MGD. Kappa statistic is a widely deployed visualization method [35] for measuring the level of agreement between outputs of two classifiers. Given two classifiers, $T_{a}$ and $T_{b}$ and an $n$-class problem, Kappa statistic $k$ is calculated from a contingency matrix of size $n \times n$. The element $m_{i, j}$ of the matrix stands for the proportion of the samples for which $T_{a}$ labels as the $i$-th class but $T_{b}$ labels as the $j$-th class. $k$ is defined as follows:

$$
\begin{aligned}
& k_{a, b}=\frac{\sum_{i} m_{i, j}-\Theta}{1-\Theta} \\
& \Theta=\sum_{i}\left(\sum_{j} m_{i, j} \times \sum_{j} m_{j, i}\right)
\end{aligned}
$$

where $\sum_{i} m_{i, j}$ is a measure of agreement, and $\Theta$ is the "agree-by-chance". $k=1$ only when the two classifiers totally agree. $k=0$ when they are independent and the agreement equals that expected by chance. It is actually more desirable when $k<0$ which means one classifier has a better chance of correcting the results than randomness when the other fails. In such case, they are “negative dependent". In General, a smaller value of $k$ indicates higher level of diversity among base classifiers.

Fig. 9 shows the Kappa diagram of MLD and MGD in two datasets. In these figures, x-axis represents the kappa statistic, and y-axis represents the average accuracy rate in each encoding matrices pair-wise. For the ensemble of $L$ classifiers, there are $(L-1) \times L / 2$ different pairs of Kappa statistical values. We evaluated the Kappa statistic and accuracy for each encoding matrix pair in MLD and MGD. The ensemble size of encoding matrices is set to 5. Fig. 9 indicates that the ECOC learners selected by MLD generally can achieve slightly higher average accuracies than MGD, but MGD can select learners with lower Kappa statistic values. Thus, as analyzed before, 
the power of MGD lies in its higher mutual diversity among the base encoding matrices, which brings the final ensemble system higher accuracies.

\subsection{Comparisons of Different Ensemble Methods}

To compare the performance of HE-ECOC with other ensemble systems, GA-ESP algorithm[45], k-TSP[43] and some tree based ensemble systems are used. Because in [45] and [43], all the datasets are standardized with the same preprocessing method as the experiments in this paper, their results listed in Table 8 are directly cited from the original papers. Besides, decision tree (DT), Random Forest (RF) and hybrid extreme rotation forest (HERF) [3] are also deployed for comparisons. For decision tree and Random Forest, the implementations in the scikit-learn library are used with the default settings [19]. The python implementation of HERF is used with the default settings (https://github.com/borjaayerdi/AdaHERF). Because the base learners of HE-ECOC are ensemble systems, for fair comparisons, although RF and HERF are already robust ensemble systems, they are still used as base learners. By this means, despite the fact that these tree based learners can deal with a multiclass problem directly, they are only used as binary classifiers in the following experiments. RF, HERF and DT are combined by OVO and OVR schemes, just as the former tow methods. To simplify the comparisons, we also list the results of the MLD and MGD based HE-ECOC using DECOC with t-test method based experimental results. As GA-ESP is implemented with SVM as base classifiers, the results of MLD and MGD are also obtained using SVM. Both k-TSP and DT are stable algorithms, and their outputs are regarded as both average and the best accuracies.

From Table 8, we can find that the MGD based HE-ECOC outperforms other algorithms with obvious advantages: it wins 5 out of 7 cases in average accuracies and 6 out of 7 cases in the best accuracies. It also obtains the highest scores in the average results across all datasets. Although the MLD based HE-ECOC method doesn't obtain such identical performances, it gets the second best results in some cases, and obtains the second highest scores in overall average performances. RF only produces the best results in the Leukemia data set. k-TSP, GA-ESP, DT and HERF never win in average and the best accuracies. Such comparisons can prove that our algorithms are of great generalization ability. 
Our D-ECOC based HE-ECOC beats other algorithms with $(N-1) \times 5$ dichotomizers. OVR based ensemble systems require $N-1$ base classifiers, and OVO based methods need $(N-1) \times N / 2$ base classifiers. So in the case that the average accuracies are close, the OVR based method have the advantage of less computational costs.

On the other hand, despite of the fail of k-TSP and GA-ESP in most cases, each of their base classifiers is trained using a gene pair in all these experiments. In this way, they can tackle the feature selection and classification tasks at the same time, and allow researchers to further explore the biological significance of the selected genes. HE-ECOC wins at the cost of a large number of genes for building base learners, so its application to the analysis of key genes is quite different from the previous methods: it mainly relies on the analysis of gene selection frequency, just as the work in [33].

\subsection{Comparisons of Different Fusion Schemes}

In the ensemble learning research field, there are many different fusion schemes for combining the final results. In former experiments, only the average scheme is used for fusion. In this subsection, some more fusion schemes are employed.

The most popular fusion method is the majority voting scheme (MV). But the original scheme can only deal with hard outputs (labels). When dealing with soft outputs (such as probabilities or distances), it needs to transform them into labels at first. Besides the average scheme, there are some other schemes employed to fuse the soft outputs directly, and the typical examples are Minimum and Maximum [29, 40]. In addition, it was found that by assigning different weights to each classifier according to its performance, the final ensemble can be more effective, as analyzed in [20]. So in the following experiments, for each base learner, its resubstitution accuracy on the training dataset is used as its weight in all weight based fusion schemes.

There are some other methods used to fuse outputs under different conditions. For example, a Naive-Bayes decision rule can calculate the estimated class conditional probabilities using the Bayes' formula, and the label with the highest posterior probability is selected as the final decision [12]. A Behavior-Knowledge Space (BKS) method evaluates all of possible combinations of class labels, which are filled in a look-up table using all data [26]. The decision templates compare a prototypical 
decision of the ensemble for the training samples in a class (the template) to the current decision of the ensemble to a test sample, so as to produce more reliable results[30]. However, since these types of methods require a very large volume of training data, they are not suitable for the analysis of microarray data. In short, in the following experiments, five fusion schemes are used for comparison purpose.

- The majority voting scheme (MV): With this method, each base classifier votes for a specific class, and the class obtaining the majority of votes is selected as the final output. Assume that for a sample $X^{*}$, the $i$-th ECOC base learner produce a label vector $\left\{d_{i 1}, d_{i 2}, \ldots, d_{i N}\right\}$, where $d_{i j}=1$ if the Hamming distance of its output for this sample is the shortest to the $j$-th class, otherwise $d_{i j}=0$. Then the final output for $X^{*}$ is shown in formula (17). When there are equal votes for more than one class, the final decision is made by randomly selecting a class from the candidates.

$$
o=\arg \max _{j=\{1, \ldots, N\}}\left(\sum_{i=1}^{N_{S}} d_{i j}\right)
$$

- The weighted majority voting scheme (WMV): As each learner obtains a special weight, the WMV scheme can break the tie of the MV scheme. Only the label obtaining the highest sum of weights is used as the final decision. Assume that the weight for the $i$-th classifier is $w_{i}$, the final decision for the sample is:

$$
o=\arg \max _{j=\{1, \ldots, N\}}\left(\sum_{i=1}^{N_{S}} w_{i} d_{i j}\right)
$$

- The minimum scheme (MIN): This scheme always uses the labels with the minimum Hamming distance as the final decisions. When there are two or more base learners achieving the same minimum distance but voting different classes, the class obtaining more votes in the ensemble wins.

$$
o=\arg \min H D_{i j, i=\left\{1, \ldots, N_{S}\right\}, j=\{1, \ldots, N\}}
$$

- The weighted minimum scheme (WMIN): By multiplying the Hamming distance by weights, the ties of the minimum scheme can be broken, and the class obtaining the minimum weighted distance wins, as shown in (20).

$$
o=\arg \min w_{i} H D_{i j, i=\left\{1, \ldots, N_{S}\right\}, j=\{1, \ldots, N\}}
$$

- The weighted average scheme (WAVE): The average of weighted Hamming distance can be 
calculated by (21), multiplying the Hamming distance by the corresponding weights. And the class with the minimum weighted average distance is treated as the final decision.

$$
o=\arg \min { }_{j=\{1, \ldots, N\}}\left(\sum_{i=1}^{N_{S}} \frac{w_{i} H D_{i j}}{N_{S}}\right)
$$

In the previous experiments, there are only 5 base learners regardless of the number of classes. So the tie in the MV scheme occurs at a high probability when the number of class is relatively small. For example, it is quite possible that for two different classes, each receives two votes individually. In such case, the MV scheme can only randomly select a class, leading to a high probability in making a wrong decision. Since the WMV scheme can break this tie, it can achieve better results in the Breast dataset compared with the MV scheme. And it is interesting to find that for the Cancers dataset, its results are completely the same as those of MV. The reason mainly lies in that the class number in the Cancers dataset is much larger than that of the Breast dataset, so the probability of two or more classes obtaining the same highest number of votes in the Cancers dataset is lower. Since it is less possible that the tie occurs, the weight doesn't play an important role as in the Breast dataset case.

For the MIN scheme, there is also a tie when two different base learners obtain the same minimum Hamming distance but vote different classes. It is solved by selecting the label receiving more votes, which is much more reliable compared with random selection. So the MIN scheme can achieve better results compared with the MV scheme. On the contrary, the WMIN scheme always selects only a single most weighted learner. But as shown in Table 2 and Table 3, the average accuracies of individual base learners are usually just around $60 \%$ in the test sets, so only using a most weighted learner can not guarantee satisfying outcomes. In general, the WMIN scheme may produce slightly worse results compared with the MIN scheme. But from Table 9, it is found that the difference between them is quite small. The overall average accuracies of the MIN scheme are higher than those of the WMIN scheme about $0.2 \%$.

On the contrary, no tie occurs in experiments when using the AVE/WAVE schemes because the average values of all distances can hardly be the same. As listed in Table 9, the average accuracies of the AVE/WAVE schemes are much higher than other fusion schemes in all cases. And the AVE/WAVE achieves the highest accuracies and average accuracies in all but one case in our experiments, and the average accuracies are about $20 \%$ higher than others'. So it can be safely 
concluded that the AVE/WAVE is the best schemes for fusing results for the HE-ECOC algorithm. In comparing the AVE and WAVE schemes, it is found that their performance is very close, and the WAVE scheme wins in most cases with slight advantages.

Fig. 10 shows the change of results with the number of base learners increasing. It is obvious that the more base learners can not guarantee better performances. For MV/WMV and MIN/WMIN, the results of both KNN and SVM change greatly with the increase of the number of base learner on the two datasets. For the Cancers dataset, the results produced by KNN drop greatly after fusing some more base learners, and recover only after ensembling more than six base learners. And those produced by SVM increase first, and then decrease after fusing more than 8 base learners. From these inconsistent results, it is impossible to predict how many base learners are required for producing the best results when using these four schemes. On the contrary, the results of AVE/WAVE are relatively stable when there are more than 4 base learners, and are all much better than those of the former four schemes. Because there is little difference among results obtained by AVE and WAVE, only the AVE scheme is used in the HE-ECOC framework for simplification.

In short, proper coding matrices are selected to construct the HE-ECOC systems with our proposed MLD and MGD schemes. So the HE-ECOC system can further improve the performance of different ECOC methods effectively by fusing only a few coding matrices. In experiments, the HE-ECOC method beats single ECOC algorithms and some other methods in almost all cases with the AVE fusion schemes.

\section{Conclusions}

In this paper, we propose a new ensemble system based on ECOC algorithms. In this ensemble scheme, different ECOC coding matrices are generated using different data-dependent ECOC algorithms. Two schemes, MLD and MGD, are designed to evaluate the diversity among coding matrices. Then highly diverse ECOC coding matrices are selected to construct a hierarchical ensemble system, called as HE-ECOC. This method can be used to solve the multi-class microarray classification problem effectively.

In our experiments, the MLD/MGD based HE-ECOC methods are tested using different feature selection methods and three data-dependent ECOC algorithms. The experimental results 
show that both MLD and MGD can successfully pick up diverse ECOC coding matrices for building ensemble system despite of different principles. And HE-ECOC is an effective method for the microarray data classification task. It usually leads to better results compared with each single ECOC method and some ensemble systems. Six different fusion schemes are applied for comparing their performances in fusing results of the HE-ECOC, and the AVE/WAVE schemes take obvious advantages over others by avoiding ties. So they are employed for the final fusion of outputs for the HE-ECOC.

Furthermore, the HE-ECOC method could be a new solution for high-dimensional classification problems. With increasing features, the multi-class classification becomes more and more intractable for many traditional methods. However, in our method, the high dimension data can help to produce different ECOC coding matrices, which eventually benefit the final ensemble system by providing diverse and accurate base learners. Thus, our HE-ECOC can be a good solution for other high-dimensional classification problems. In addition, because ECOC methods focus on the division of classes, HE-ECOC may reveal the similar function of oncogenes in different cancer subtypes when dealing with multi-class microarray data. It will be our future research direction.

\section{Acknowledgments}

This work is supported by National Science Foundation of China (61100106, 61174161, 61273303 and 61303080). 
Parameters:

$N E$ : the number of coding matrices needed to be produced in the scheme; $N L$ : the number of coding matrices needed to be selected for ensemble system; $T$ : training data; $f_{i}$ : the $i$-th feature subset; $C_{i}$ : the $i$-th coding matrix.

for $i=1$ to $N E$ do

Use a FS method to produce $f_{i}$ based on $T$ with an increasing step;

Generate $C_{i}$ using an ECOC algorithm based on $f_{i}$;

end

for $i=1$ to $N E-1$ do

for $j=1$ to $N E$ do

Calculate $P D_{i, j}$ according to Formula (9);

end

Fig. 1. Pseudo code of the MLD scheme

Parameters:

$A$ : the number of ECOC algorithms used; $C_{i, j}$ : the $i$-th coding matrix produced by the $j$-th

ECOC algorithm;

for $i=1$ to $A$ do

for $j=1$ to $A$ do

Use a FS method to produce $f_{j}$ based on $T$;

Generate $C_{i}^{j}$ using $i$-th ECOC algorithm based on $f_{j}$;

end

end

for $i=1$ to $N E-1$ do

for $j=2$ to $N E$ do

Calculate $P D_{i, j}$ according to Formula (9);

end

Fig. 2. Pseudo code of the MGD scheme 


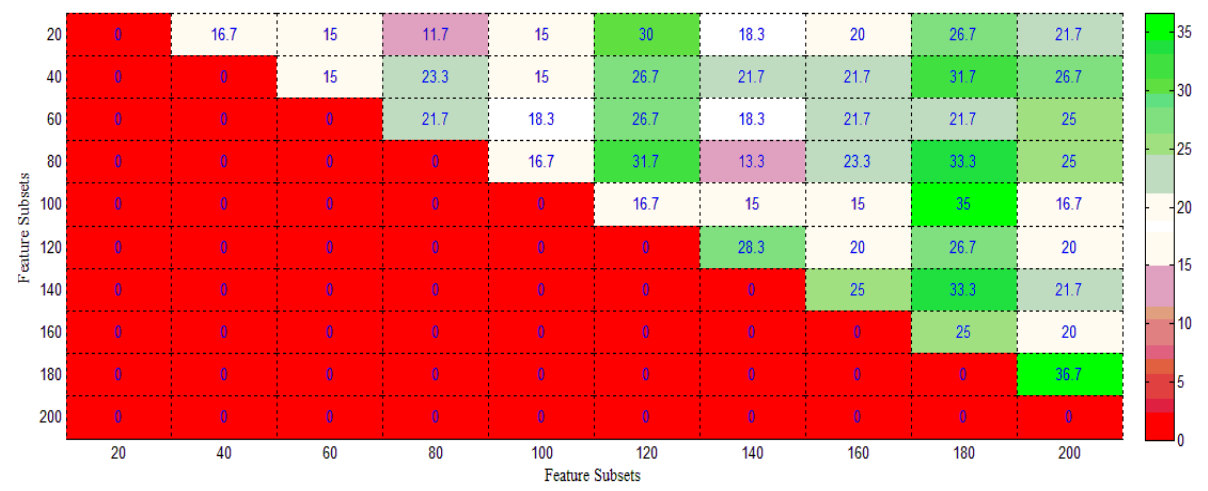

Fig. 3. An example of $P D$ score matrix.
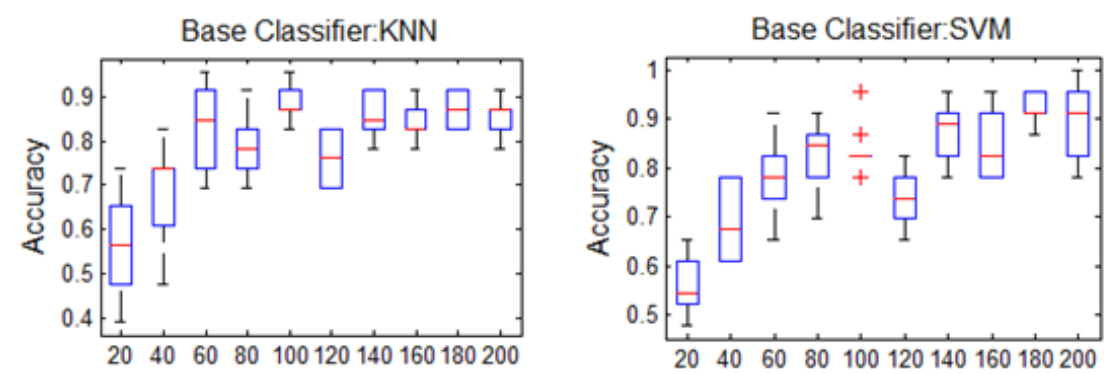

(a)
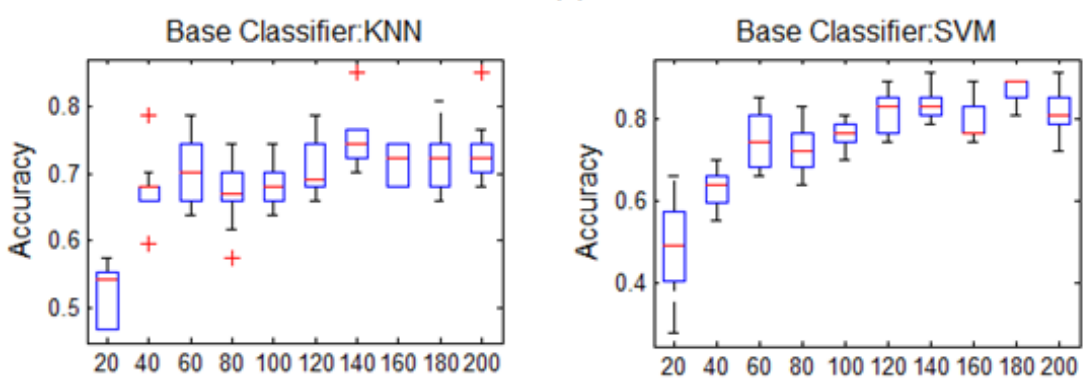

(b)

Fig. 4. Classification accuracies obtained by ECOC-One with increasing size of feature subsets using Laplacian Score on: (a) the Breast dataset; (b) the Cancers dataset. 


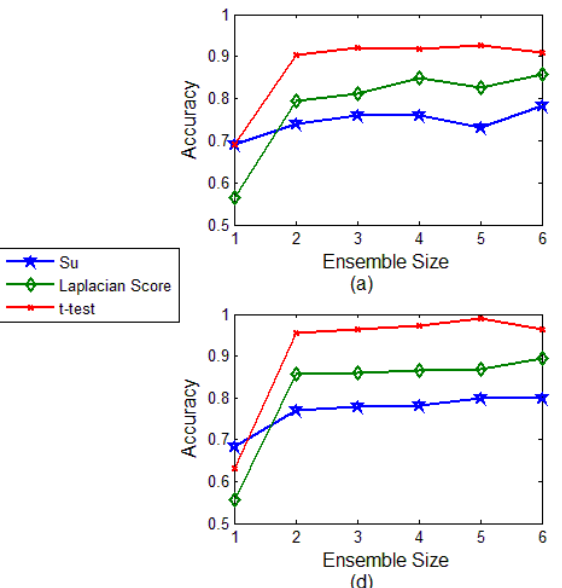

(d)

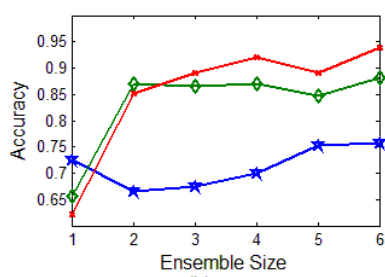

(b)

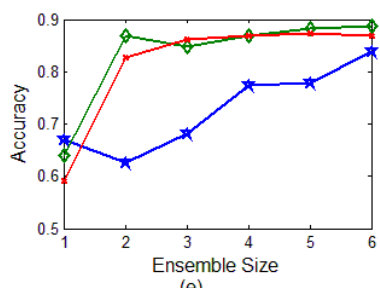

(e)

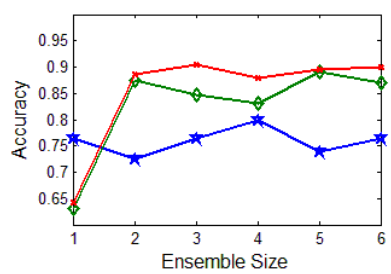

(c)

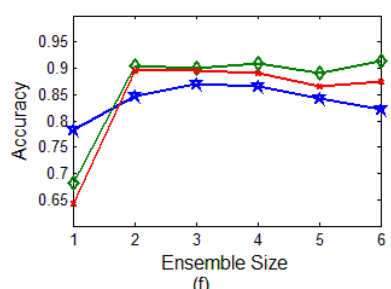

Fig. 5. Typical results of classification accuracy obtained by MLD based HE-ECOC with increasing ensemble size for the Breast dataset: (a) ECOC-One with KNN; (b) DECOC with KNN;(c) Forest-ECOC with KNN;(d) ECOC-One with SVM;(e) DECOC with SVM; (f) Forest-ECOC with SVM;
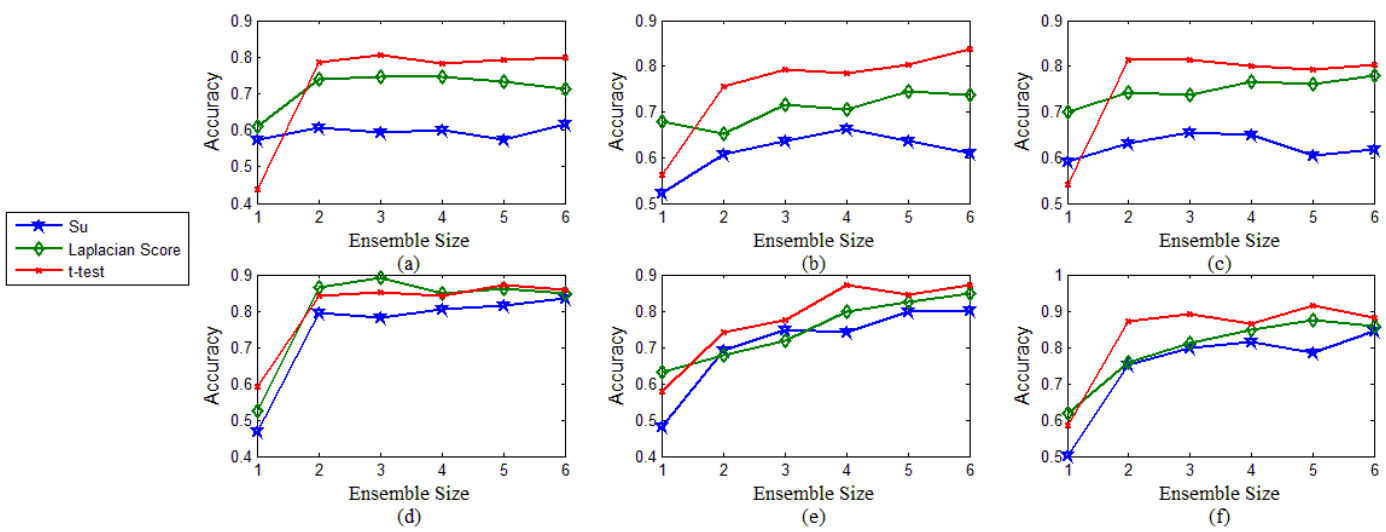

Fig. 6. Typical results of classification accuracy obtained by MLD based HE-ECOC with increasing ensemble size for the Cancers dataset: (a) ECOC-One with KNN;(b)DECOC with KNN;(c)Forest-ECOC with KNN;(d) ECOC-One with SVM; (e) DECOC with SVM;(f) Forest-ECOC with SVM; 

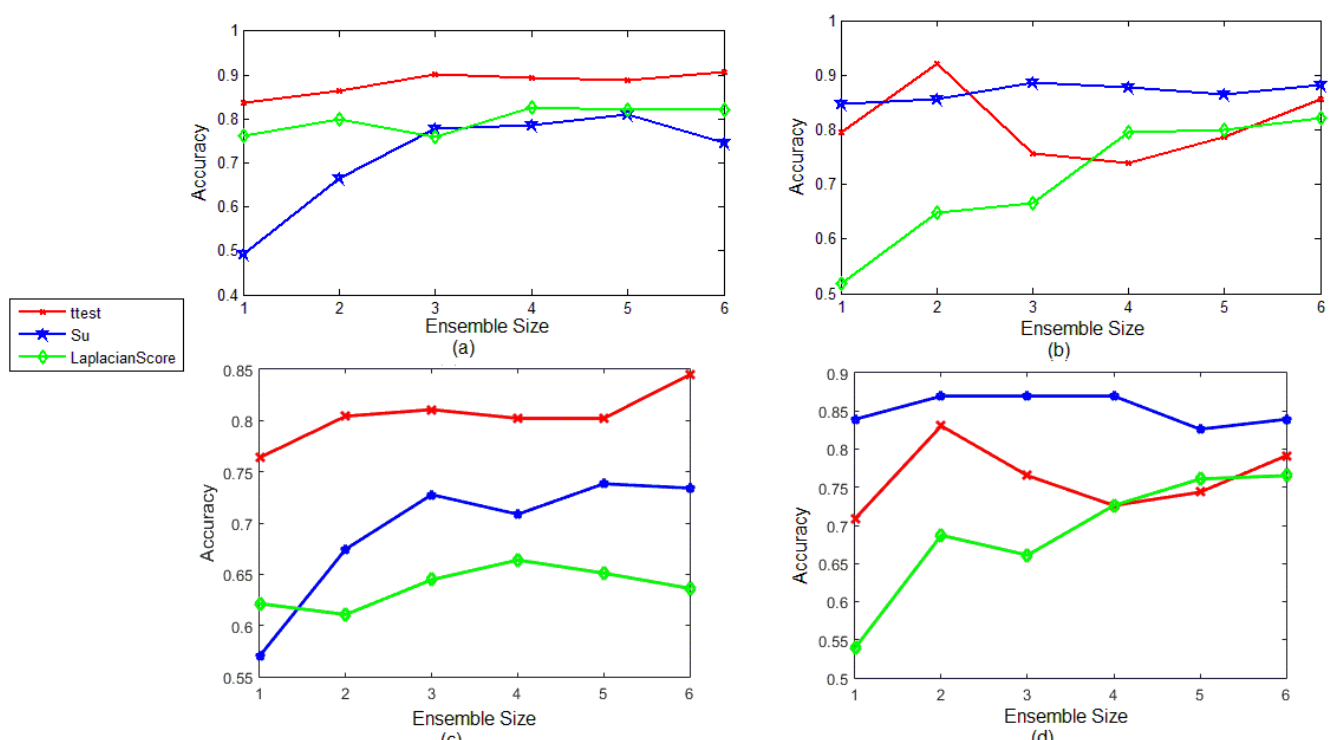

Fig. 7. Typical results of classification accuracy obtained by MGD based HE-ECOC with increasing ensemble size: (a) the Breast dataset with KNN; (b) the Breast dataset with SVM; (c) the Cancers dataset with KNN; (d) the Cancers dataset with SVM;
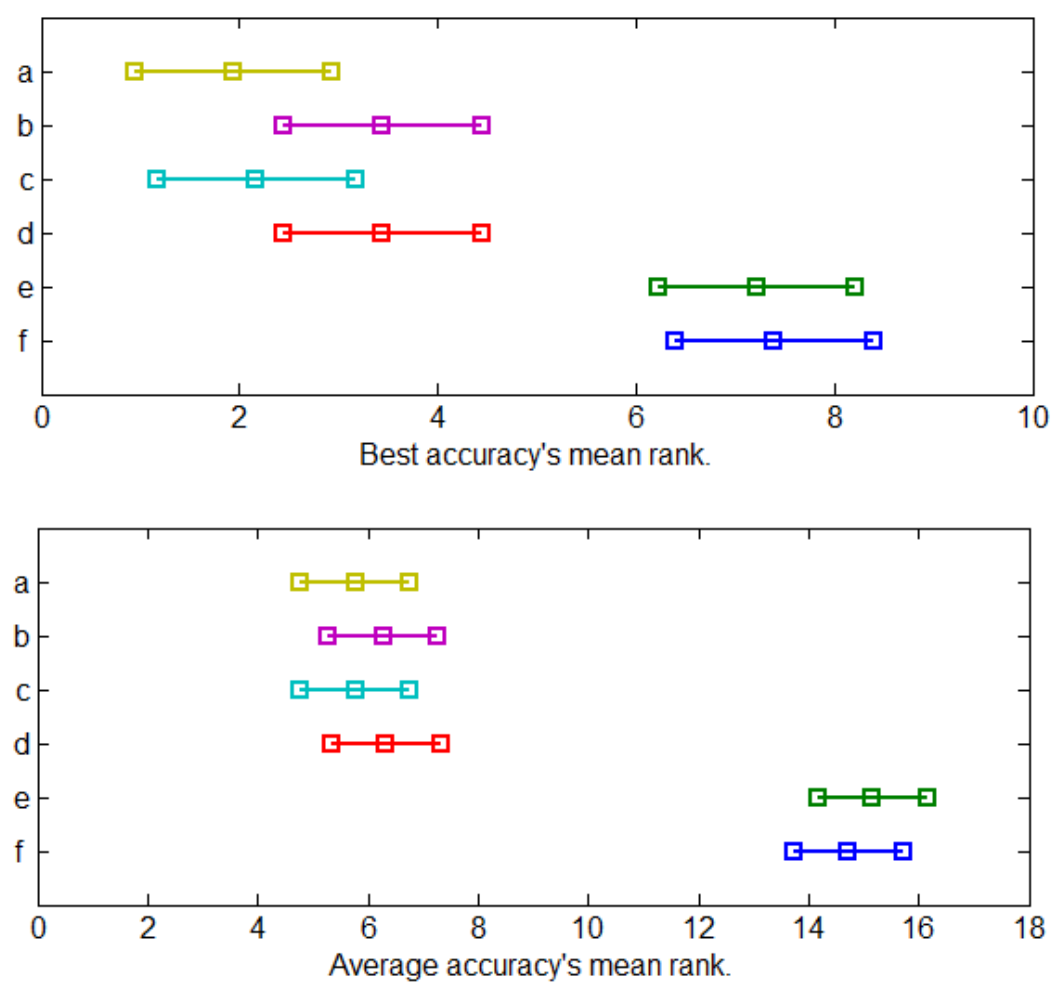

Fig. 8. The comparison results of rival methods based on the Nemenyi test: (a) MGD with SVM; (b) MGD with KNN; (c) MLD with SVM; (d) MLD with KNN; (e) Single ECOC with SVM; (f) Single ECOC with KNN. 

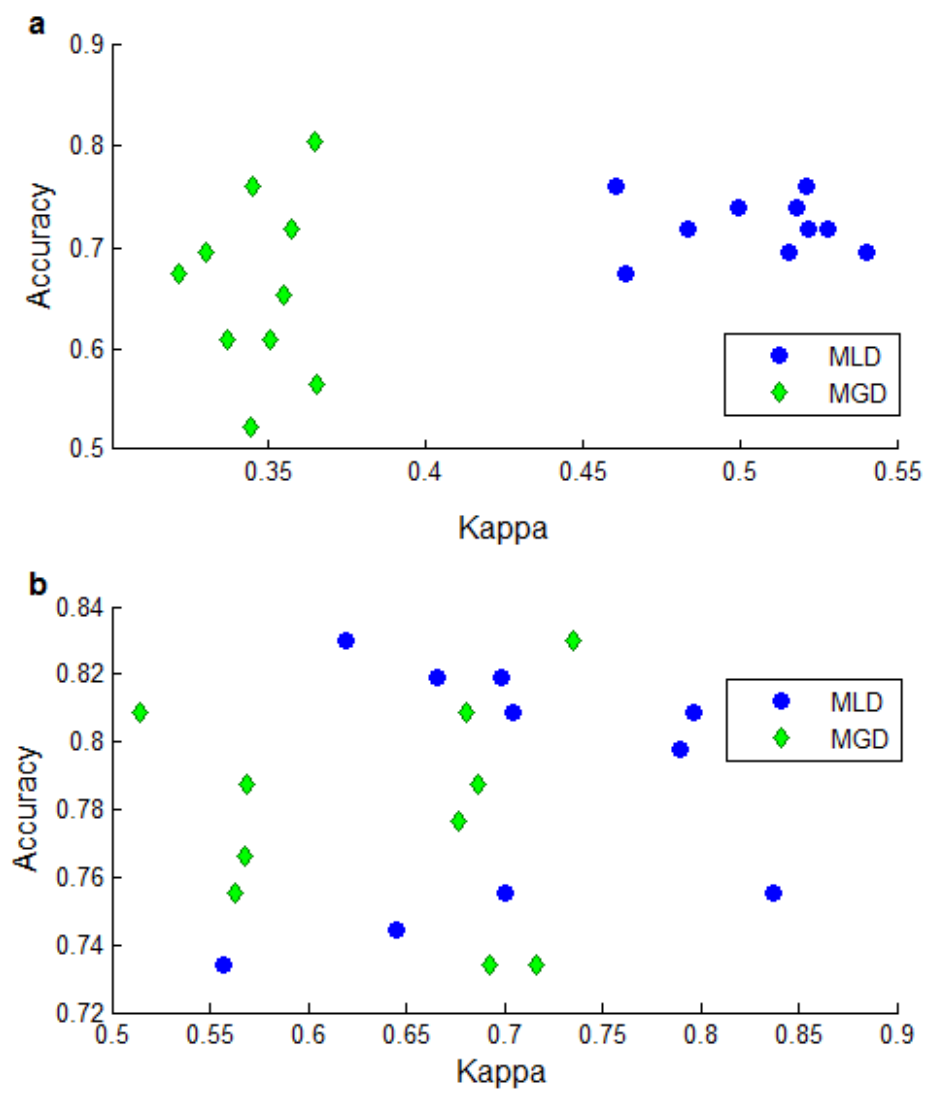

Fig. 9. Kappa diagrams of MLD and MGD: (a) Breast. (b) Cancers. 

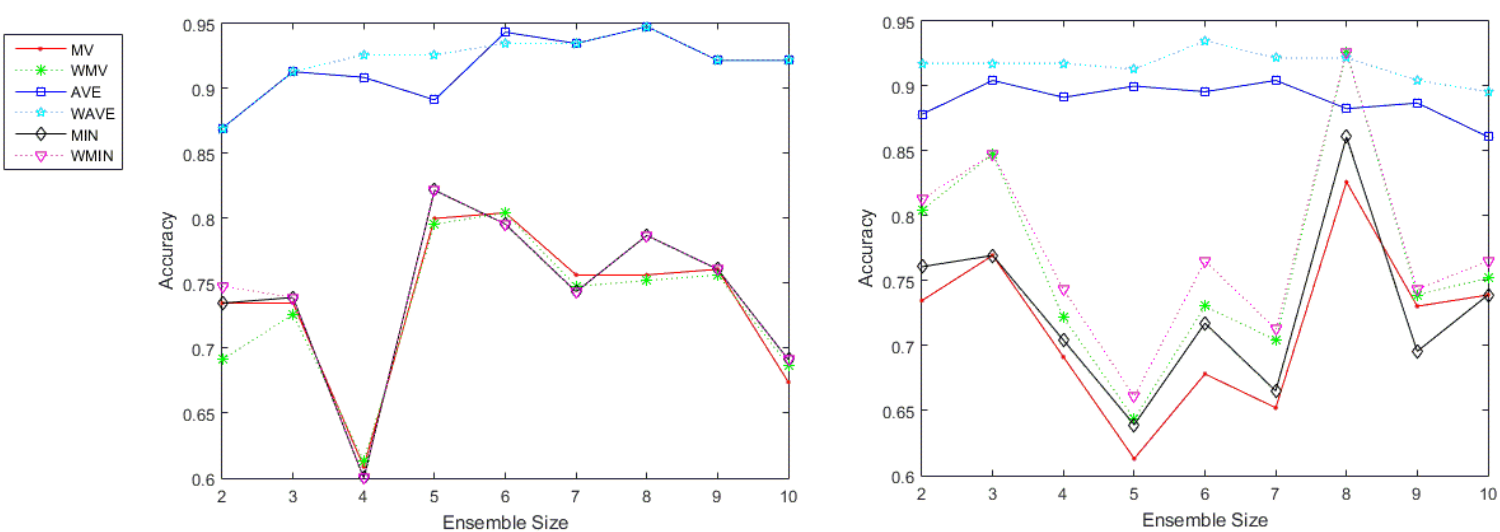

(a)

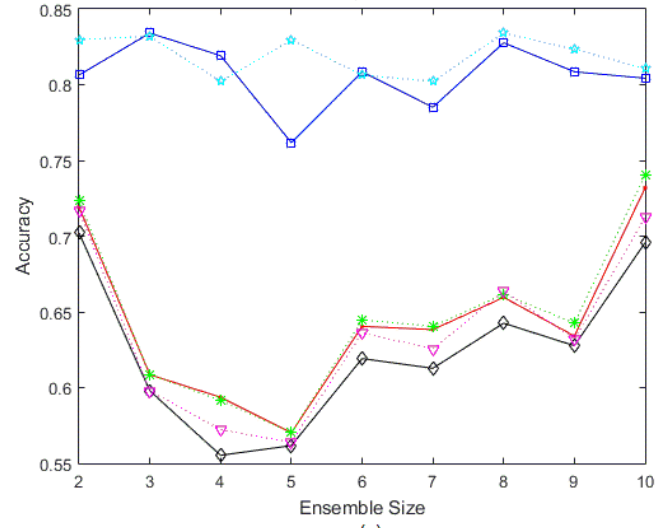

(b)

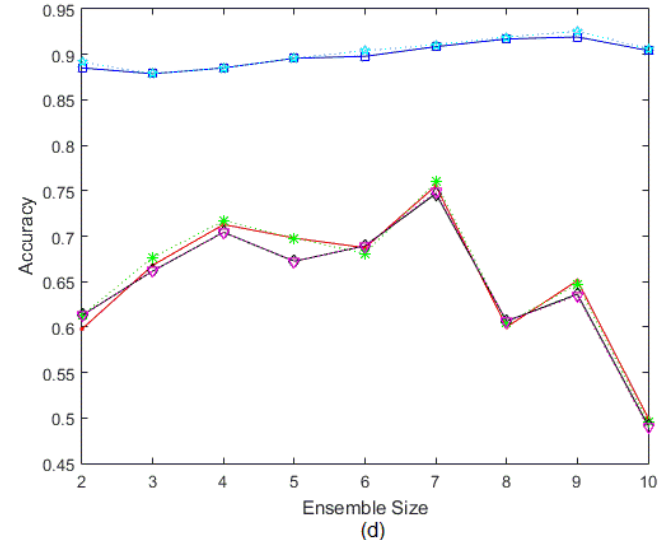

Fig. 10. Typical results of different fusion schemes obtained by MLD (Forest-ECOC) based HE-ECOC with increasing ensemble size using Laplacian Score: (a) the Breast dataset with KNN; (b) the Breast dataset with SVM; (c) the Cancers dataset with KNN; (d) the Cancers dataset with SVM. 
Table 1. Multi-class datasets used in experiments

\begin{tabular}{llccc}
\hline Dataset & No. of classes & No. of genes & No. of training samples & No. of test samples \\
\hline Leukaemia & 3 & 7129 & 38 & 34 \\
DLBCL & 6 & 4026 & 58 & 30 \\
Lung & 3 & 7129 & 64 & 32 \\
SRBCT & 4 & 2308 & 63 & 20 \\
Breast & 5 & 9216 & 54 & 30 \\
Cancers & 11 & 12,533 & 100 & 74 \\
GCM & 14 & 16,063 & 144 & 46 \\
\hline
\end{tabular}

Table 2. The comparison of average and best classification accuracies among individual ECOC coding matrices and different ECOC ensemble method for the Breast dataset (Ensemble Size=5).

\begin{tabular}{|c|c|c|c|c|c|c|c|}
\hline \multirow{2}{*}{\multicolumn{2}{|c|}{ Base Classifier: KNN }} & \multicolumn{2}{|c|}{$\mathrm{Su}$} & \multicolumn{2}{|c|}{ Laplacian Score } & \multicolumn{2}{|c|}{ t-test } \\
\hline & & $A_{v g}$ & $\mathrm{~A}_{\text {best }}$ & $A_{\text {avg }}$ & $A_{\text {best }}$ & $A_{\text {avg }}$ & $\mathrm{A}_{\text {best }}$ \\
\hline \multicolumn{2}{|c|}{ (a)ECOC-One } & $56.52 \pm 12.91$ & 73.91 & $69.13 \pm 14.75$ & 82.61 & $69.13 \pm 12.35$ & 78.26 \\
\hline \multicolumn{2}{|c|}{ (b)DECOC } & $65.65 \pm 11.61$ & 78.26 & $72.61 \pm 7.17$ & 78.26 & $62.17 \pm 9.51$ & 73.91 \\
\hline \multicolumn{2}{|c|}{ (c)Forest-ECOC } & $63.04 \pm 12.47$ & 73.91 & $76.52 \pm 8.39$ & 82.61 & $64.35 \pm 15.42$ & 82.61 \\
\hline \multirow{3}{*}{ MLD } & (d)ECOC-One & $73.04 \pm 9.79$ & 86.96 & $82.61 \pm 9.67$ & 100.00 & $92.61 \pm 7.13$ & 100.00 \\
\hline & (e)DECOC & $75.22 \pm 12.21$ & 82.61 & $84.78 \pm 7.26$ & 91.30 & $89.13 \pm 8.25$ & 95.65 \\
\hline & (f)Forest-ECOC & $73.91 \pm 9.42$ & 82.61 & $89.13 \pm 8.30$ & 95.65 & $89.57 \pm 9.31$ & 95.65 \\
\hline \multicolumn{2}{|c|}{ (g)MGD } & $78.26 \pm 9.72$ & 86.96 & $83.91 \pm 10.21$ & 91.30 & $87.39 \pm 9.21$ & 95.65 \\
\hline
\end{tabular}




\begin{tabular}{|c|c|c|c|c|c|c|c|}
\hline \multirow{2}{*}{\multicolumn{2}{|c|}{ Base Classifier: SVM }} & \multicolumn{2}{|c|}{$\mathrm{Su}$} & \multicolumn{2}{|c|}{ Laplacian Score } & \multicolumn{2}{|c|}{ t-test } \\
\hline & & $\mathrm{A}_{\text {avg }}$ & $\mathrm{A}_{\text {best }}$ & $A_{\text {avg }}$ & $A_{\text {best }}$ & $A_{\text {avg }}$ & $A_{\text {best }}$ \\
\hline \multicolumn{2}{|c|}{ (a)ECOC-One } & $55.65 \pm 13.29$ & 65.22 & $68.26 \pm 7.59$ & 78.26 & $63.04 \pm 12.10$ & 82.61 \\
\hline \multicolumn{2}{|c|}{ (b)DECOC } & $63.91 \pm 9.25$ & 69.57 & $66.96 \pm 6.39$ & 73.91 & $59.13 \pm 9.93$ & 73.91 \\
\hline \multicolumn{2}{|c|}{ (c)Forest-ECOC } & $68.26 \pm 9.30$ & 73.91 & $78.26 \pm 7.84$ & 86.96 & $64.35 \pm 10.08$ & 82.61 \\
\hline \multirow{3}{*}{ MLD } & (d)ECOC-One & $80.00 \pm 10.55$ & 91.30 & $86.96 \pm 6.08$ & 91.30 & $99.13 \pm 0.83$ & 100.00 \\
\hline & (e)DECOC & $77.83 \pm 12.31$ & 86.96 & $88.26 \pm 4.21$ & 91.30 & $87.39 \pm 11.27$ & 95.65 \\
\hline & (f)Forest-ECOC & $84.35 \pm 13.64$ & 95.65 & $89.13 \pm 7.43$ & 100.00 & $86.52 \pm 7.47$ & 91.30 \\
\hline \multicolumn{2}{|c|}{ (g)MGD } & $77.83 \pm 12.52$ & 91.30 & $86.09 \pm 10.12$ & 91.30 & $99.13 \pm 0.83$ & 100.00 \\
\hline
\end{tabular}


Table 3. The comparison of average and best classification accuracies among individual ECOC coding matrices and different ECOC ensemble method for the Cancers dataset (Ensemble Size=5).

\begin{tabular}{|c|c|c|c|c|c|c|c|}
\hline \multirow{2}{*}{\multicolumn{2}{|c|}{ Base Classifier: KNN }} & \multicolumn{2}{|c|}{$\mathrm{Su}$} & \multicolumn{2}{|c|}{ Laplacian Score } & \multicolumn{2}{|c|}{ t-test } \\
\hline & & $\mathrm{A}_{\mathrm{avg}}$ & $A_{\text {best }}$ & $A_{\text {avg }}$ & $A_{\text {best }}$ & $\mathrm{A}_{\text {avg }}$ & $A_{\text {best }}$ \\
\hline \multicolumn{2}{|c|}{ (a)ECOC-One } & $52.13 \pm 4.18$ & 57.45 & $57.23 \pm 10.38$ & 63.83 & $59.15 \pm 10.47$ & 68.09 \\
\hline \multicolumn{2}{|c|}{ (b)DECOC } & $67.87 \pm 7.23$ & 78.72 & $61.06 \pm 5.19$ & 68.09 & $70.00 \pm 4.15$ & 76.60 \\
\hline \multicolumn{2}{|c|}{ (c)Forest-ECOC } & $56.17 \pm 4.40$ & 65.96 & $43.83 \pm 12.18$ & 51.06 & $54.04 \pm 8.49$ & 63.83 \\
\hline \multirow{3}{*}{ MLD } & (d)ECOC-One & $57.23 \pm 3.30$ & 65.96 & $73.40 \pm 9.16$ & 78.72 & $79.15 \pm 7.06$ & 80.85 \\
\hline & (e)DECOC & $63.62 \pm .5 .31$ & 72.34 & $74.47 \pm 8.10$ & 78.72 & $80.43 \pm 9.16$ & 87.23 \\
\hline & (f)Forest-ECOC & $60.43 \pm 7.29$ & 74.47 & $76.17 \pm 10.43$ & 89.36 & $79.36 \pm 9.82$ & 87.23 \\
\hline \multicolumn{2}{|c|}{ (g)MGD } & $65.96 \pm 8.35$ & 74.47 & $74.04 \pm 9.15$ & 80.85 & $78.09 \pm 8.18$ & 82.98 \\
\hline \multirow{2}{*}{\multicolumn{2}{|c|}{ Base Classifier: SVM }} & \multicolumn{2}{|c|}{$\mathrm{Su}$} & \multicolumn{2}{|c|}{ Laplacian Score } & \multicolumn{2}{|c|}{ t-test } \\
\hline & & $A_{\text {avg }}$ & $\mathrm{A}_{\text {best }}$ & $\mathrm{A}_{\text {avg }}$ & $A_{\text {best }}$ & $A_{\text {avg }}$ & $A_{\text {best }}$ \\
\hline \multicolumn{2}{|c|}{ (a)ECOC-One } & $48.30 \pm 12.49$ & 65.96 & $46.81 \pm 7.33$ & 55.32 & $50.21 \pm 12.63$ & 65.96 \\
\hline \multicolumn{2}{|c|}{ (b)DECOC } & $63.19 \pm 13.87$ & 70.21 & $52.34 \pm 6.19$ & 59.57 & $61.70 \pm 15.46$ & 76.60 \\
\hline \multicolumn{2}{|c|}{ (c)Forest-ECOC } & $57.87 \pm 7.42$ & 61.70 & $59.15 \pm 7.24$ & 48.94 & $58.51 \pm 9.44$ & 65.96 \\
\hline \multirow{3}{*}{ MLD } & (d)ECOC-One & $81.49 \pm 8.17$ & 87.23 & $86.17 \pm 10.51$ & 93.62 & $87.23 \pm 9.27$ & 95.74 \\
\hline & (e)DECOC & $79.79 \pm 9.63$ & 89.36 & $82.55 \pm 9.13$ & 87.23 & $84.47 \pm 7.61$ & 89.36 \\
\hline & (f)Forest-ECOC & $78.51 \pm 7.39$ & 85.11 & $87.45 \pm 9.58$ & 95.74 & $91.49 \pm 6.86$ & 95.74 \\
\hline \multicolumn{2}{|c|}{ (g)MGD } & $83.40 \pm 10.54$ & 89.36 & $85.96 \pm 9.43$ & 93.62 & $87.23 \pm 9.13$ & 93.62 \\
\hline
\end{tabular}


Table 4. Comparisons of accuracies on different datasets using D-ECOC.

\begin{tabular}{|c|c|c|c|c|c|c|}
\hline \multirow{2}{*}{ Dataset } & \multicolumn{2}{|c|}{ Single ECOC } & \multicolumn{2}{c|}{ MLD } & \multicolumn{2}{c|}{ MGD } \\
\cline { 2 - 7 } & $\mathrm{A}_{\text {avg }}$ & $\mathrm{A}_{\text {best }}$ & $\mathrm{A}_{\text {avg }}$ & $\mathrm{A}_{\text {best }}$ & $\mathrm{A}_{\text {avg }}$ & $\mathrm{A}_{\text {best }}$ \\
\hline SRBCT & $88.26 \pm 9.13$ & 95.65 & $\mathbf{9 8 . 7 0} \pm \mathbf{1 . 8 1}$ & $\mathbf{1 0 0}$ & $96.09 \pm 3.15$ & $\mathbf{1 0 0}$ \\
\hline Lung & $88.98 \pm 8.64$ & 93.22 & $96.10 \pm 1.75$ & 98.31 & $\mathbf{9 7 . 2 9} \pm \mathbf{1 . 1 6}$ & $\mathbf{1 0 0}$ \\
\hline DLBCL & $76.52 \pm 6.97$ & 86.96 & $95.42 \pm 0.04$ & 96.61 & $\mathbf{9 5 . 9 3} \pm \mathbf{2 . 7 5}$ & $\mathbf{9 8 . 3 1}$ \\
\hline GCM & $56.11 \pm 1.97$ & 57.41 & $63.62 \pm 7.38$ & 72.34 & $\mathbf{6 5 . 9 6} \pm \mathbf{1 3 . 2 6}$ & $\mathbf{7 4 . 4 7}$ \\
\hline Leukaemia & $61.47 \pm 3.31$ & 66.32 & $78.51 \pm 9.66$ & 85.11 & $\mathbf{7 9 . 7 9} \pm 13.33$ & $\mathbf{8 9 . 3 6}$ \\
\hline
\end{tabular}

Table 5. Comparisons of accuracies on different datasets using Forest-ECOC.

\begin{tabular}{|c|c|c|c|c|c|c|}
\hline \multirow{2}{*}{ Dataset } & \multicolumn{2}{|c|}{ Single ECOC } & \multicolumn{2}{c|}{ MLD } & \multicolumn{2}{c|}{ MGD } \\
\cline { 2 - 7 } & $\mathrm{A}_{\text {avg }}$ & $\mathrm{A}_{\text {best }}$ & $\mathrm{A}_{\text {avg }}$ & $\mathrm{A}_{\text {best }}$ & $\mathrm{A}_{\text {avg }}$ & $\mathrm{A}_{\text {best }}$ \\
\hline SRBCT & $90.87 \pm 3.44$ & 95.65 & $96.52 \pm 3.38$ & $\mathbf{1 0 0}$ & $\mathbf{9 8 . 7 0 \pm 1 . 5 1}$ & $\mathbf{1 0 0}$ \\
\hline Lung & $89.83 \pm 7.06$ & 94.92 & $\mathbf{9 6 . 9 5} \pm \mathbf{5 . 7 7}$ & $\mathbf{1 0 0}$ & $95.59 \pm 0.80$ & 96.91 \\
\hline DLBCL & $77.39 \pm 9.99$ & 86.96 & $92.17 \pm 1.83$ & 95.65 & $\mathbf{9 5 . 2 2} \pm 7.33$ & $\mathbf{1 0 0}$ \\
\hline GCM & $\mathbf{4 8 . 3 3} \pm \mathbf{9 . 1 5}$ & $\mathbf{5 5 . 5 6}$ & $46.30 \pm 3.25$ & 51.85 & $40.74 \pm 1.57$ & 42.59 \\
\hline Leukaemia & $62.63 \pm 6.30$ & 68.42 & $73.58 \pm 5.74$ & $\mathbf{8 3 . 1 6}$ & $\mathbf{7 7 . 8 9} \pm 9.61$ & 82.26 \\
\hline
\end{tabular}

Table 6. Best accuracy's mean rank.

\begin{tabular}{|c|c|c|c|c|c|c|}
\hline \multirow{2}{*}{ Dataset } & \multicolumn{3}{|c|}{ KNN } & \multicolumn{3}{c|}{ SVM } \\
\cline { 2 - 7 } & Single ECOC & MLD & MGD & Single ECOC & MLD & MGD \\
\hline Breast & 6 & 2.78 & 3 & 6.44 & 2.44 & 2.33 \\
\hline Cancers & 9.56 & 4.89 & 4.67 & 8.78 & 2.67 & 2.33 \\
\hline Avg. Rank & 7.78 & 3.84 & 3.84 & 7.61 & 2.56 & 2.33 \\
\hline
\end{tabular}

Table 7. Average accuracy's mean rank.

\begin{tabular}{|c|c|c|c|c|c|c|}
\hline \multirow{2}{*}{ Dataset } & \multicolumn{3}{|c|}{ KNN } & \multicolumn{3}{c|}{ SVM } \\
\cline { 2 - 7 } & Single ECOC & MLD & MGD & Single ECOC & MLD & MGD \\
\hline Breast & 15 & 6 & 6 & 15.11 & 6.33 & 7.33 \\
\hline
\end{tabular}




\begin{tabular}{|c|c|c|c|c|c|c|}
\hline Cancers & 15.22 & 7.44 & 7.33 & 16 & 6 & 5 \\
\hline Avg. Rank & 15.11 & 6.72 & 6.67 & 15.56 & 6.17 & 6.17 \\
\hline
\end{tabular}


Table 8. The comparisons among HE-ECOC and other ensemble systems on different multi-class datasets

\begin{tabular}{|c|c|c|c|c|c|c|c|c|c|c|c|c|c|c|c|c|c|c|c|c|}
\hline \multirow[t]{2}{*}{ Dataset } & \multicolumn{2}{|c|}{ GA-ESP-OVO } & \multicolumn{2}{|c|}{ GA-ESP-OVR } & \multirow{2}{*}{$\begin{array}{l}\text { k-TSP } \\
\text { OVO }\end{array}$} & \multirow{2}{*}{$\begin{array}{l}\text { k-TSP } \\
\text { OVR }\end{array}$} & \multirow{2}{*}{$\begin{array}{c}\text { DT-O } \\
\text { vo }\end{array}$} & \multirow{2}{*}{$\begin{array}{c}\text { DT-O } \\
\text { VR }\end{array}$} & \multicolumn{2}{|c|}{ HERF-OVO } & \multicolumn{2}{|c|}{ HERF-OVR } & \multicolumn{2}{|c|}{ RF-OVO } & \multicolumn{2}{|c|}{ RF-OVR } & \multicolumn{2}{|c|}{ MLD } & \multicolumn{2}{|c|}{ MGD } \\
\hline & $\mathrm{A}_{\mathrm{avg}}$ & $\mathrm{A}_{\text {best }}$ & $\mathrm{A}_{\mathrm{avg}}$ & $\mathrm{A}_{\text {best }}$ & & & & & $\mathrm{A}_{\text {avg }}$ & $\mathrm{A}_{\text {best }}$ & $\mathrm{A}_{\text {avg }}$ & $\mathrm{A}_{\text {best }}$ & $\mathrm{A}_{\mathrm{avg}}$ & $\mathrm{A}_{\text {best }}$ & $\mathrm{A}_{\text {avg }}$ & $A_{\text {best }}$ & $\mathrm{A}_{\mathrm{avg}}$ & $\mathrm{A}_{\text {best }}$ & $\overline{A_{\text {avg }}}$ & $\mathrm{A}_{\text {best }}$ \\
\hline Breast & 0.820 & 0.867 & 0.803 & 0.867 & 0.800 & 0.733 & 0.867 & 0.800 & 0.774 & 0.867 & 0.884 & 0.957 & 0.785 & 0.867 & 0.865 & 0.957 & 0.874 & 0.957 & 0.942 & 1.00 \\
\hline Cancers & 0.739 & 0.797 & 0.419 & 0.366 & 0.635 & 0.662 & 0.622 & 0.622 & 0.728 & 0.797 & 0.645 & 0.716 & 0.661 & 0.716 & 0.632 & 0.716 & 0.845 & 0.894 & 0.853 & 0.936 \\
\hline SRBCT & 0.840 & 0.900 & 0.630 & 0.800 & 0.950 & 0.840 & 0.950 & 0.800 & 0.833 & 0.900 & 0.856 & 0.900 & 0.820 & 0.900 & 0.815 & 0.900 & 0.987 & 1.00 & 0.961 & 1.00 \\
\hline Lung & 0.936 & 0.940 & 0.907 & 0.925 & 0.940 & 0.940 & 0.851 & 0.910 & 0.910 & 0.940 & 0.945 & 0.983 & 0.887 & 0.940 & 0.931 & 0.983 & 0.961 & 0.983 & 0.973 & 1.00 \\
\hline DLBCL & 0.957 & 0.967 & 0.617 & 0.633 & 0.833 & 0.957 & 0.733 & 0.800 & 0.913 & 0.933 & 0.941 & 0.967 & 0.828 & 0.933 & 0.932 & 0.967 & 0.954 & 0.967 & 0.959 & 0.983 \\
\hline GCM & 0.650 & 0.696 & 0.430 & 0.478 & 0.544 & 0.650 & 0.348 & 0.457 & 0.503 & 0.543 & 0.523 & 0.543 & 0.410 & 0.543 & 0.523 & 0.543 & 0.636 & 0.723 & 0.660 & 0.745 \\
\hline Leukemia & 0.942 & 0.955 & 0.727 & 0.768 & 0.912 & 0.942 & 0.912 & 0.912 & 0.893 & 0.955 & 0.898 & 0.942 & 0.947 & 0.955 & 0.878 & 0.955 & 0.785 & 0.851 & 0.798 & 0.894 \\
\hline Average & 0.841 & 0.875 & 0.648 & 0.691 & 0.802 & 0.818 & 0.755 & 0.757 & 0.793 & 0.848 & 0.813 & 0.858 & 0.763 & 0.836 & 0.797 & 0.860 & 0.863 & 0.911 & 0.867 & 0.937 \\
\hline
\end{tabular}


Table 9. The comparison of average and best classification accuracies among individual ECOC coding matrices and different ECOC ensemble method for the Breast and the cancer datasets (Ensemble Size=5) with t-test feature selection.

\begin{tabular}{|c|c|c|c|c|c|c|c|c|c|c|c|c|c|c|c|}
\hline \multirow{2}{*}{ Data } & \multirow{2}{*}{\multicolumn{3}{|c|}{ Methods }} & \multicolumn{2}{|c|}{ MV } & \multicolumn{2}{|c|}{ WMV } & \multicolumn{2}{|c|}{ MIN } & \multicolumn{2}{|c|}{ WMIN } & \multicolumn{2}{|c|}{ AVE } & \multicolumn{2}{|c|}{ WAVE } \\
\hline & & & & $A_{v g}$ & $\mathrm{~A}_{\text {best }}$ & $\mathrm{A}_{\mathrm{avg}}$ & $A_{\text {best }}$ & $A_{\text {avg }}$ & $A_{\text {best }}$ & $A_{\text {avg }}$ & $A_{\text {best }}$ & $\mathrm{A}_{\text {avg }}$ & $\mathrm{A}_{\text {best }}$ & $A_{\text {avg }}$ & $A_{\text {best }}$ \\
\hline \multirow{9}{*}{ Breast } & \multirow{4}{*}{ KNN } & \multirow{3}{*}{ MLD } & ECOC-One & $65.27 \pm 18.18$ & 82.61 & $65.65 \pm 20.88$ & 82.61 & $66.09 \pm 20.74$ & 82.61 & $66.09 \pm 13.14$ & 82.61 & $92.61 \pm 7.13$ & 100.00 & $93.13 \pm 6.18$ & 100.00 \\
\hline & & & DECOC & $73.04 \pm 6.16$ & 78.26 & $73.48 \pm 15.68$ & 82.61 & $72.61 \pm 17.47$ & 86.96 & $72.61 \pm 16.57$ & 86.96 & $89.13 \pm 8.25$ & 95.65 & $89.13 \pm 7.22$ & 95.65 \\
\hline & & & Forest-ECOC & $75.48 . \pm 8.15$ & 82.61 & $83.91 \pm 7.15$ & 86.96 & $83.04 \pm 13.13$ & 86.96 & $82.61 \pm 13.24$ & 86.96 & $89.57 \pm 9.31$ & 95.65 & $90.45 \pm 6.31$ & 95.65 \\
\hline & & & MGD & $66.09 \pm 10.18$ & 78.26 & $66.09 \pm 15.18$ & 78.26 & $67.39 \pm 19.19$ & 78.26 & $66.96 \pm 16.39$ & 78.26 & $87.39 \pm 9.21$ & 95.65 & $88.62 \pm 3.37$ & 95.65 \\
\hline & \multirow{4}{*}{ SVM } & \multirow{3}{*}{ MLD } & ECOC-One & $52.17 \pm 15.24$ & 78.26 & $52.17 \pm 21.24$ & 78.26 & $55.66 \pm 21.29$ & 78.26 & $55.66 \pm 22.22$ & 78.26 & $99.13 \pm 0.83$ & 100.00 & $99.23 \pm 0.32$ & 100.00 \\
\hline & & & DECOC & $67.39 \pm 10.22$ & 78.26 & $66.52 \pm 18.21$ & 82.60 & $65.65 \pm 22.47$ & 86.96 & $65.65 \pm 21.72$ & 86.96 & $87.39 \pm 11.27$ & 95.65 & $87.89 \pm 9.24$ & 95.65 \\
\hline & & & Forest-ECOC & $84.34 . \pm 6.14$ & 86.96 & $84.35 \pm 6.15$ & 86.96 & $85.65 \pm 4.11$ & 86.96 & $84.78 \pm 12.65$ & 86.96 & $86.52 \pm 7.47$ & 91.30 & $86.52 \pm 7.71$ & 91.30 \\
\hline & & & MGD & $68.26 \pm 8.19$ & 78.26 & $70.87 \pm 10.20$ & 78.26 & $67.82 \pm 13.17$ & 78.26 & $67.83 \pm 19.77$ & 78.26 & $99.13 \pm 0.83$ & 100.00 & $99.13 \pm 0.83$ & 100.00 \\
\hline & \multicolumn{3}{|c|}{ Average } & $\begin{array}{c}65.37 \pm \\
10.31 \\
\end{array}$ & 80.44 & $\begin{array}{c}70.38 \pm \\
14.34 \\
\end{array}$ & 82.07 & $\begin{array}{c}70.49 \pm \\
16.45 \\
\end{array}$ & 83.15 & $\begin{array}{c}70.27 \pm \\
16.96 \\
\end{array}$ & 83.15 & $91.36 \pm 6.79$ & 96.74 & $\begin{array}{c}91.76 \pm \\
5.15 \\
\end{array}$ & 96.74 \\
\hline \multirow{9}{*}{ Cancers } & \multirow{4}{*}{ KNN } & \multirow{3}{*}{ MLD } & ECOC-One & $58.51 \pm 8.29$ & 82.98 & $58.51 \pm 8.29$ & 82.98 & $59.57 \pm 8.09$ & 82.98 & $60.21 \pm 8.69$ & 87.23 & $79.15 \pm 7.06$ & 80.85 & $79.15 \pm 3.56$ & 80.85 \\
\hline & & & DECOC & $68.30 \pm 8.54$ & 82.98 & $68.30 \pm 8.54$ & 82.98 & $68.51 \pm 8.77$ & 85.11 & $68.72 \pm 8.22$ & 85.11 & $80.43 \pm 9.16$ & 87.23 & $80.32 \pm 8.61$ & 87.23 \\
\hline & & & Forest-ECOC & $66.59 \pm 8.35$ & 85.11 & $66.59 \pm 8.35$ & 85.11 & $66.17 \pm 9.11$ & 82.98 & $65.53 \pm 8.07$ & 74.47 & $79.36 \pm 9.82$ & 87.23 & $79.36 \pm 8.75$ & 87.23 \\
\hline & & & MGD & $60.21 \pm 9.82$ & 80.85 & $60.21 \pm 9.82$ & 80.85 & $60.00 \pm 10.09$ & 82.98 & $60.21 \pm 8.92$ & 76.60 & $78.09 \pm 8.18$ & 82.98 & $78.21 \pm 9.08$ & 82.98 \\
\hline & \multirow{4}{*}{ SVM } & \multirow{3}{*}{ MLD } & ECOC-One & $70.64 \pm 8.97$ & 87.23 & $70.64 \pm 8.97$ & 87.23 & $71.70 \pm 8.90$ & 87.23 & $71.70 \pm 9.55$ & 87.23 & $87.23 \pm 9.27$ & 95.74 & $87.23 \pm 9.27$ & 95.74 \\
\hline & & & DECOC & $55.53 \pm 9.91$ & 87.23 & $55.53 \pm 9.91$ & 87.23 & $54.89 \pm 11.23$ & 89.36 & $55.95 \pm 10.16$ & 87.23 & $84.47 \pm 7.61$ & 89.36 & $84.47 \pm 7.61$ & 89.36 \\
\hline & & & Forest-ECOC & $61.45 \pm 9.30$ & 87.23 & $61.45 \pm 9.30$ & 87.23 & $61.92 \pm 9.33$ & 87.23 & $61.06 \pm 8.33$ & 78.72 & $91.49 \pm 6.86$ & 95.74 & $91.49 \pm 6.86$ & 95.74 \\
\hline & & & MGD & $61.92 \pm 8.87$ & 87.23 & $61.92 \pm 8.87$ & 87.23 & $63.19 \pm 9.59$ & 89.36 & $61.28 \pm 8.45$ & 85.11 & $87.23 \pm 9.13$ & 93.62 & $87.23 \pm 9.13$ & 93.62 \\
\hline & \multicolumn{3}{|c|}{ Average } & $62.89 \pm 9.01$ & 85.11 & $62.89 \pm 9.01$ & 85.11 & $63.24 \pm 9.39$ & 85.90 & $63.08 \pm 8.80$ & 82.71 & $\mathbf{8 3 . 4 3} \pm 8.39$ & 89.09 & $83.43 \pm 7.86$ & 89.09 \\
\hline
\end{tabular}




\section{References}

[1] E. L. Allwein, R. E. Schapire, and Y. Singer, "Reducing multiclass to binary: A unifying approach for margin classifiers," The Journal of Machine Learning Research, vol. 1, pp. 113-141, 2001.

[2] U. Alon, N. Barkai, D. A. Notterman, K. Gish, S. Ybarra, D. Mack, et al., "Broad patterns of gene expression revealed by clustering analysis of tumor and normal colon tissues probed by oligonucleotide arrays," Proceedings of the National Academy of Sciences of the United States of America, vol. 96, pp. 6745-6750, Jun 81999.

[3] M. G. B. Ayerdi, "Hybrid extreme rotation forest," Neural Netw., vol. 52, pp. 33-42, 2014.

[4] M. A. Bagheri, G. A. Montazer, and E. Kabir, "A subspace approach to error correcting output codes," Pattern Recognition Letters, vol. 34, pp. 176-184, 2013.

[5] M. Á. Bautista, S. Escalera, X. Baró, P. Radeva, J. Vitriá, and O. Pujol, "Minimal design of error-correcting output codes," Pattern Recognition Letters, vol. 33, pp. 693-702, 2012.

[6] M. Á. Bautista, S. Escalera, X. Baró, and O. Pujol, "On the design of an ECOC-Compliant Genetic Algorithm," Pattern Recognition, vol. 47, pp. 865-884, 2014.

[7] A. Ben-Dor, L. Bryhn, N. Friedman, I. nachman, M. Schum-mer, and Z. Yakhini, "Tissue classification with gene expression profiles," Journal of Computational Biology, vol. 4, pp. 290 - 2301, 2000.

[8] V. Bolón-Canedoa, N. Sánchez-Maroño, A. Alonso-Betanzos, J. M. Benítez, and F. Herrer, "A review of microarray datasets and applied feature selection methods," Information Sciences, vol. 282, pp. 111-135, 2014.

[9] C. C. Chang and C. J. Lin, "LIBSVM: a library for support vector machines," ACM Transactions on Intelligent Systems and Technology, vol. 2, p. 27, 2011.

[10] K. Cramme and Y. Singer, "On the learnability and design of output codes for multiclass problems," Machine Learning, vol. 47, pp. 201-233, 2002.

[11] C. Ding and H. Peng, "Minimum Redundancy Feature Selection from Microarray Gene Expression Data," Proceedings of the Computational Systems Bioinformatics, pp. 523 - 529, 2003.

[12] P. Domingos and M. Pazzani, "On the Optimality of the Simple Bayesian Classifier under Zero-One Loss," Machine Learning, vol. 29, pp. 103-130, 1997.

[13] L. O. E. Tapia, P. Bulacio, and L. Angelone, "Multiclass classification of microarray data samples with a reduced number of genes," Bmc Bioinformatics, vol. 12, p. 59, 2011.

[14] S. Escalera, O. Pujol, and P. Radeva, "ECOC-ONE: A novel coding and decoding strategy," International Conference on Pattern Recognition, IEEE Computer Society, vol. 3, pp. 578-581, 2006.

[15] S. Escalera, O. Pujol, and P. Radeva, "Boosted Landmarks of Contextual Descriptors and Forest-ECOC: A novel framework to detect and classify objects in cluttered scenes," Pattern Recognition Letters, vol. 28, pp. 1759-1768, 2007.

[16] S. Escalera, D. M. Tax, O. Pujol, P. Radeva, and R. P. Duin, "Subclass problem-dependent design for error-correcting output codes," IEEE Transactions on attern Analysis and Machine Intelligence vol. 30, pp. 1041-1054, 2008.

[17] S. Escalera, O. Pujol, and P. Radeva, "On the decoding process in ternary error-correcting output codes," IEEE Transactions on Pattern Analysis and Machine Intelligence, vol. 32, pp. 120-134, 2010.

[18] S. Escalera, O. Pujol, and P. Radeva, "Error-Correcting Ouput Codes Library," J. Mach. Learn. Res., vol. 11, pp. 661-664, 2010.

[19] e. a. Fabian Pedregosa, "Scikit-learn: Machine Learning in Python," JMLR 12, vol. 12, pp. 2825-2830, 2011.

[20] G. Fumera and F. Roli, "A theoretical and experimental analysis of linear combiners for multiple classifier systems," leee Transactions on Pattern Analysis and Machine Intelligence, vol. 27, pp. 942-956, Jun 2005.

[21] N. García-Pedrajas and C. Fyfe, "Evolving output codes for multiclass problems," IEEE Transactions on Evolutionary Computation, vol. 12, pp. 93-106, 2008.

[22] T. Golub, D. Slonim, P. Tamayo, C. Huard, M. Gaasenbeek, J. Mesirov, et al., "Molecular classification of cancer: class discovery and class prediction by gene expression monitoring," Science, vol. 286, pp. 531 537, 1999.

[23] W. R. Hamming, "Error detecting and error correcting codes," Bell System Technical Journal, vol. 29, pp. 147-160, 1950.

[24] X. He, D. Cai, and P. Niyogi, "Laplacian score for feature selection," NIPS, vol. 186, 2005.

[25] Z. Q. Hong and J. Y. Yang, "Optimal Discriminant Plane for a Small Number of Samples and Design Method of Classifier on the Plane," Pattern Recognition, vol. 24, pp. 317-324, 1991.

[26] Y. S. Huang and S. C.Y., "Combination of multiple experts for the recognition of unconstrained handwritten numerals," IEEE Trans. on Pattern Analysis and Machine Intelligence, vol. 17, pp. 90-94, 1995. 
[27] J. Khan, J. Wei, M. Ringner, L. Saal, M. Ladanyi, F. West-ermann, et al., "Classification and diagnostic prediction of cancers using gene expression profiling and artificial neural networks," Nature Medicine, vol. 7, pp. $673-679,2001$.

[28] E. B. Kong and T. G. Dietterich, "Error-Correcting Output Coding Corrects Bias and Variance," Proceedings of the Twelfth International Conference on Machine Learning, pp. 313-321, 1995.

[29] L. I. Kuncheva, "An application of OWA operators to the aggregation of multiple classication decisions," In The Ordered Weighted Averaging operators. Theory and Applications, vol. Kluwer Academic Publisher, USA, pp. 330-343, 1997.

[30] L. I. Kuncheva, J. C. Bezdek, and R. P. W. Duin, "Decision templates for multiple classifier fusion: an experimental comparison," Pattern Recognition, vol. 34, pp. 299-314, 2001.

[31] L. I. Kuncheva, "Combining pattern classifiers: methods and algorithms," Wiley, 2004.

[32] K. Liu, M. Tong, S. Xie, and V. T. Y. Ng, "Genetic Programming Based Ensemble System for Microarray Data Classification," Computational and Mathematical Methods in Medicine, vol. 2015, Article ID 193406, 2015.

[33] K. H. Liu and C. G. Xu, "A genetic programming-based approach to the classification of multiclass microarray datasets," Bioinformatics, vol. 25, pp. 331-337, 2009.

[34] A. C. Lorena and A. C. Carvalho, "Evolutionary design of multiclass support vector machines," Journal of Intelligent and Fuzzy Systems, vol. 18, pp. 445-454, 2007.

[35] D. D. Margineantu and T. G. Dietterich, "Pruning adaptive boosting," Proc. 14th International Conference on Machine Learning, pp. 211-218, 1997.

[36] F. Masulli and G. Valentini, "Effectiveness of error correcting output codes in multiclass learning problems," Multiple Classifier Systems, pp. 107-116, 2000.

[37] C. M. Perou, T. Sørlie, M. B. Eisen, M. v. d. Rijn, S. S. Jeffrey, C. a. Rees, et al., "Molecular portraits of human breast tumours," Nature, vol. 406, pp. 747-752, 2000.

[38] O. Pujol, P. Radeva, and J. Vitria, "Discriminant ecoc: A heuristic method for application dependent design of error correcting output codes," IEEE Transactions on Pattern Analysis and Machine Intelligence, vol. 28, pp. 1007-1012, 2006.

[39] S. Ramaswamy, R. R. P. Tamayo, and e. al., "Multi-class cancer diagnosis using tumor gene expression signatures," Proc Natl Acad Sci, vol. 98, pp. 15149-15154, 2001.

[40] R. E. Schapire, "The strenght of weak learnability," Machine Learning, vol. 5, pp. 197-227, 1990.

[41] M. A. Shipp and e. al., "Diffuse Large B-cell Lymphoma Outcome Prediction by Gene-expression Profiling and Supervised Machine Learning," Nature Medicine, vol. 8, pp. 68-74, 2002.

[42] A. I. Su, J. B. Welsh, L. M. Sapinoso, S. G. Kern, P. Dimitrov, H. Lapp, et al., "Molecular classification of human carcinomas by use of gene expression signatures," Cancer research, vol. 61, pp. 7388-7393, 2001.

[43] A. Tan, D. Naiman, L. Xu, R. Winslow, and D. Geman, "Simple decision rules for classifying human cancers from gene expression profiles," Bioinformatics, vol. 21, pp. 3896-3904, 2005.

[44] E. Tapia, P. Bulacio, and L. Angelone, "Recursive ECOC classification," Pattern Recognition Letters, vol. 31, pp. 210-215, 2010.

[45] M. Tong, K. Liu, C. Xu, and W. Ju, "An ensemble of SVM classifiers based on gene pairs," Computers in Biology and Medicine, vol. 43, pp. 729-737, 2013.

[46] L. J. van't Veer, H. Y. Dai, M. J. van de Vijver, Y. D. D. He, A. A. M. Hart, M. Mao, et al., "Gene expression profiling predicts clinical outcome of breast cancer," Nature, vol. 415, pp. 530-536, Jan 312002.

[47] M. West, "Bayesian Factor Regression Models in the 'Large p, Small n' Paradigm," Bayesian Statistics, vol. 7, pp. 723-732, 2003.

[48] H. L. Yu, S. Gao, B. Qin, and J. Zhao, "Multiclass microarray data classification based on confidence evaluation," Genetics and Molecular Research, vol. 11, pp. 1357-1369, 2012.

[49] L. Yu and H. Liu, "Efficient feature selection via analysis of relevance and redundancy," Journal of Machine Learning Research, vol. 5, pp. 1205-1224, Oct 2004. 